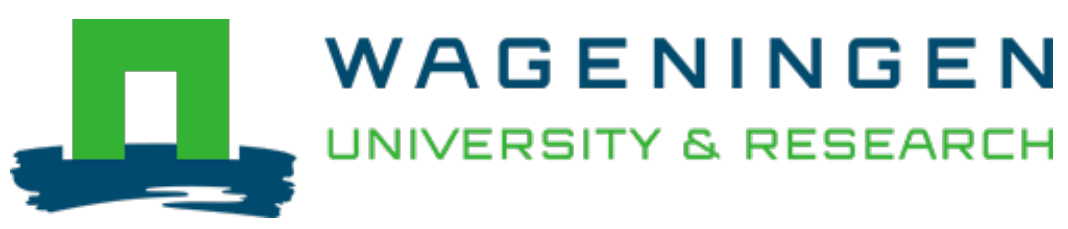

\title{
Design principles of a minimal auxin response system
}

Nature Plants

Kato, Hirotaka; Mutte, Sumanth K.; Suzuki, Hidemasa; Crespo, Isidro; Das, Shubhajit et al https://doi.org/10.1038/s41477-020-0662-y

This article is made publicly available in the institutional repository of Wageningen University and Research, under the terms of article $25 \mathrm{fa}$ of the Dutch Copyright Act, also known as the Amendment Taverne. This has been done with explicit consent by the author.

Article 25 fa states that the author of a short scientific work funded either wholly or partially by Dutch public funds is entitled to make that work publicly available for no consideration following a reasonable period of time after the work was first published, provided that clear reference is made to the source of the first publication of the work.

This publication is distributed under The Association of Universities in the Netherlands (VSNU) 'Article $25 \mathrm{fa}$ implementation' project. In this project research outputs of researchers employed by Dutch Universities that comply with the legal requirements of Article $25 \mathrm{fa}$ of the Dutch Copyright Act are distributed online and free of cost or other barriers in institutional repositories. Research outputs are distributed six months after their first online publication in the original published version and with proper attribution to the source of the original publication.

You are permitted to download and use the publication for personal purposes. All rights remain with the author(s) and / or copyright owner(s) of this work. Any use of the publication or parts of it other than authorised under article $25 \mathrm{fa}$ of the Dutch Copyright act is prohibited. Wageningen University \& Research and the author(s) of this publication shall not be held responsible or liable for any damages resulting from your (re)use of this publication.

For questions regarding the public availability of this article please contact openscience.library@wur.nl 


\title{
Design principles of a minimal auxin response system
}

\author{
Hirotaka Kato ${ }^{1,2}$, Sumanth K. Mutte $\mathbb{1}^{1,6}$, Hidemasa Suzuki, ${ }^{3,6}$, Isidro Crespo ${ }^{4,6}$, Shubhajit Das ${ }^{1,6}$, \\ Tatyana Radoeva ${ }^{1,6}$, Mattia Fontana ${ }^{1,5,6}$, Yoshihiro Yoshitake ${ }^{3}$, Emi Hainiwa3 ${ }^{3}$ Willy van den Berg ${ }^{1}$, \\ Simon Lindhoud ${ }^{1}$, Kimitsune Ishizaki ${ }^{\circledR}{ }^{2}$, Johannes Hohlbein ${ }^{1}{ }^{5}$, Jan Willem Borst ${ }^{1}$, D. Roeland Boer ${ }^{4}{ }^{4}$, \\ Ryuichi Nishihama $\mathbb{B}^{3}$, Takayuki Kohchi $\mathbb{B}^{3}$ and Dolf Weijers $\mathbb{B}^{1 凶}$
}

\begin{abstract}
Auxin controls numerous growth processes in land plants through a gene expression system that modulates ARF transcription factor activity ${ }^{1-3}$. Gene duplications in families encoding auxin response components have generated tremendous complexity in most land plants, and neofunctionalization enabled various unique response outputs during development $^{1,3,4}$. However, it is unclear what fundamental biochemical principles underlie this complex response system. By studying the minimal system in Marchantia polymorpha, we derive an intuitive and simple model where a single auxin-dependent A-ARF activates gene expression. It is antagonized by an auxin-independent B-ARF that represses common target genes. The expression patterns of both ARF proteins define developmental zones where auxin response is permitted, quantitatively tuned or prevented. This fundamental design probably represents the ancestral system and formed the basis for inflated, complex systems.
\end{abstract}

The plant hormone auxin controls essentially all aspects of growth and development, and developmental contexts determine its many unique responses ${ }^{1,2}$. TIR1/AFB F-box proteins perceive auxin and promote the ubiquitination and degradation of Aux/IAA transcriptional repressors. Aux/IAAs inhibit DNA-binding ARF transcription factors through direct interaction, and auxin thus releases ARFs from inhibition ${ }^{3}$. Although this signalling module seems simple, each component is encoded by a large gene family in most land plants, particularly in vascular plants ${ }^{4,5}$, allowing overwhelming combinatorial interaction complexity (Fig. 1a; 6 TIR1/AFBs, 29 Aux/IAAs and 23 ARFs; $>4,000$ combinations in Arabidopsis thaliana). Given different biochemical properties of family members, sets of response components can trigger unique local responses ${ }^{1,3}$, contributing to the paradoxical functional diversity of the chemically simple auxin hormone. Genetic and functional studies in flowering plants suggest functional interactions and trends in diversification among the many ARFs. ARFs are phylogenetically placed into deeply conserved $\mathrm{A} / \mathrm{B} / \mathrm{C}$ classes $^{4-6}$. A-ARFs are considered transcriptional activators, and some B- and C-ARFs are considered repressors ${ }^{7}$. Systems-wide interaction analysis among Arabidopsis Aux/IAAs and ARFs suggests more prominent auxin regulation of A-ARFs than B/C-ARFs ${ }^{8,9}$, and individual A- and B-ARFs in the moss Physcomitrella patens can antagonize through competition for DNA sites ${ }^{10}$. However, there are several counterexamples where A-ARFs directly repress targets ${ }^{11}$, Aux/IAAs interact with $\mathrm{B} / \mathrm{C}-\mathrm{ARFs}^{8,9}$ and $\mathrm{A}$ - and $\mathrm{B}-\mathrm{ARF}$ bind different DNA sequences ${ }^{12}$. Because each gene in a multigene family may have sub- or neofunctionalized during evolution, it is entirely unclear what basic biochemical architecture underlies the auxin response system. Recently, we and others have reconstructed the evolutionary history of auxin response components and found that the irreducible complexity in early-diverging land plants encompasses one TIR1/AFB receptor, one Aux/IAA and three ARFs $(\mathrm{A} / \mathrm{B} / \mathrm{C})^{4,5,13,14}$. The extant liverwort Marchantia polymorpha is representative of this minimal system (Fig. 1a) ${ }^{15,16}$ and may thus resemble the ancestral system before acquiring complexity. Here we used the M. polymorpha auxin response system to understand a minimal auxin response system.

Given that functional diversification in M. polymorpha is by definition restricted to the ARF family, we first determined whether the three M. polymorpha ARFs (A, MpARF1 (Mp1g12750); B, MpARF2 (Mp4g11820); C, MpARF3 (Mp1g07070)) have unique functions. MpARF1 is a major mediator of auxin-dependent transcription, as mutation causes strong developmental phenotypes, auxin insensitivity and the loss of auxin-dependent transcription ${ }^{17}$. An Mparf3 mutant also shows developmental defects, but no evident changes in auxin response $\mathrm{e}^{4,5}$. When expressed from the MpARF1 promoter, neither MpARF2 nor MpARF3 complemented developmental defects in the Mparf1 mutant (Fig. 1b,c), despite comparable expression levels (Extended Data Fig. 1a), which suggests non-equivalent ARF functions.

ARFs have a typical domain topology with a DNA-binding domain (DBD), a middle region (MR) and a Phox/Bem1 (PB1) oligomerization domain (Fig. 1d). To investigate the functional diversification among MpARFs, we performed domain swaps (Fig. 1e) and expressed chimaeric proteins from the MpARF1 promoter in the Mparf1 mutant background. ARF111 (each domain derived from MpARF1) fully complemented Mparf1 defects (Fig. If and Extended Data Fig. 1b), suggesting that linkers between domains did not affect function. We first compared DBD functions: ARF211 mostly rescued the growth and developmental defects of the Mparf1 mutant, producing flat thalli with normal gemmae (Fig. If and Extended Data Fig. 1) and a mildly impaired auxin response (Fig. 1f). Additional thalli were formed in an upside-down direction and produced rhizoids towards the air. In contrast, ARF311 hardly complemented thallus growth and did not produce flat thalli

'Laboratory of Biochemistry, Wageningen University, Wageningen, The Netherlands. ${ }^{2}$ Graduate School of Science, Kobe University, Kobe, Japan. ${ }^{3}$ Graduate School of Biostudies, Kyoto University, Kyoto, Japan. ${ }^{4}$ Alba Synchrotron, Cerdanyola del Vallès, Barcelona, Spain. ${ }^{5}$ Laboratory of Biophysics, Wageningen University, Wageningen, The Netherlands. ${ }^{6}$ These authors contributed equally: Sumanth K. Mutte, Hidemasa Suzuki, Isidro Crespo, Shubhajit Das, Tatyana Radoeva, Mattia Fontana. 凶e-mail: dolf.weijers@wur.nl 


\begin{tabular}{|c|c|c|}
\hline & At & $M p$ \\
\hline TIR1/AFB & 6 & 1 \\
\hline$\frac{\text { AuxIn }}{\text { بـ }}$ & & \\
\hline Aux/IAA & 29 & 1 \\
\hline $\begin{array}{l}\text { ARF } \\
\downarrow \downarrow\end{array}$ & 23 & 3 \\
\hline
\end{tabular}

b

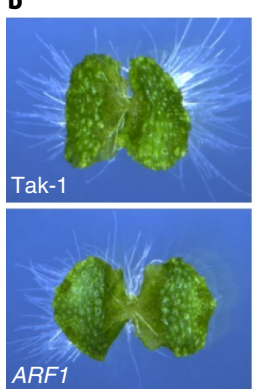

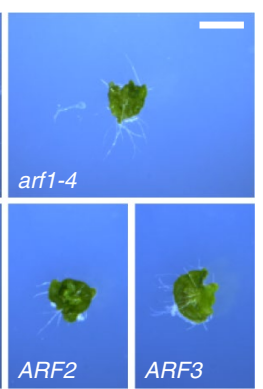

200 amino acids

d

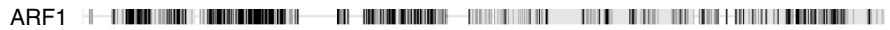

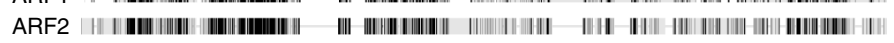

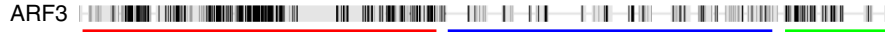
DBD

f

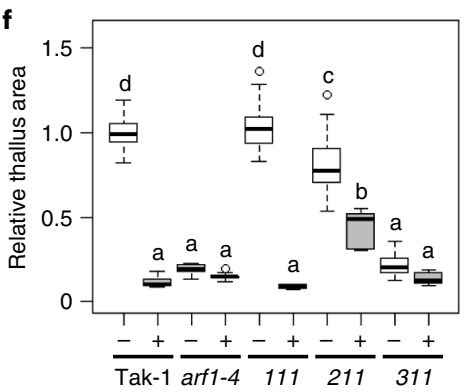

g

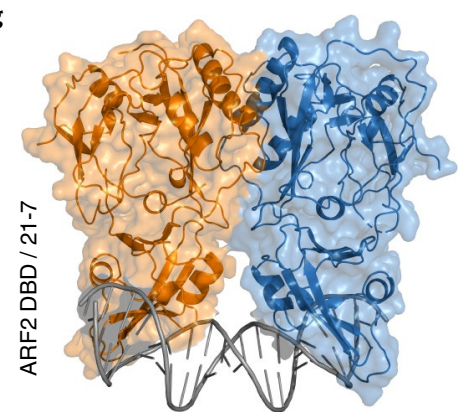

h

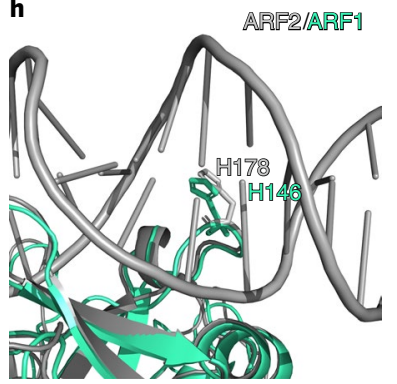

i

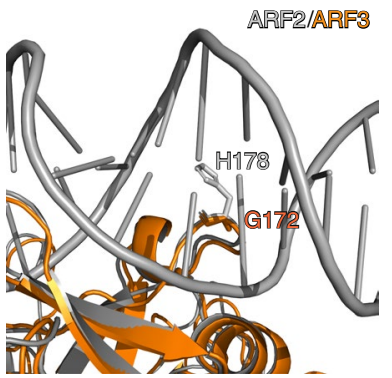

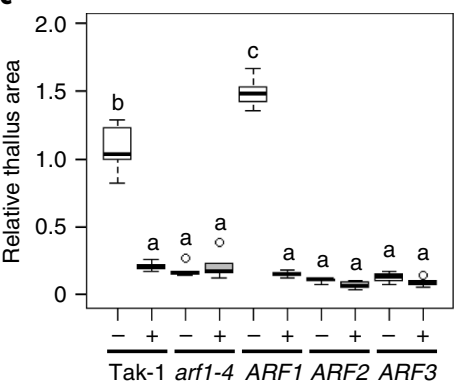

e

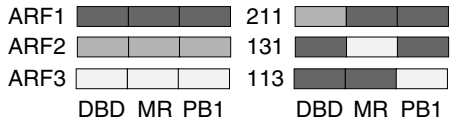

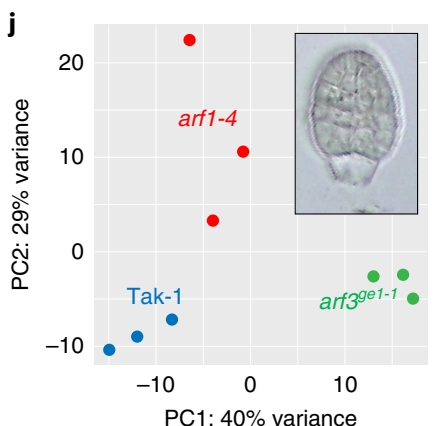

$\mathbf{k}$

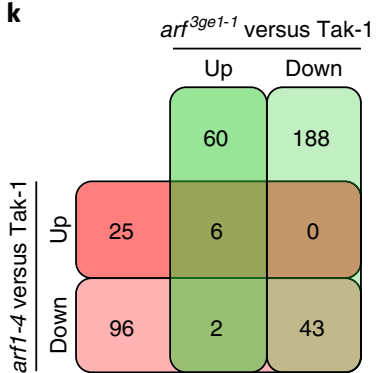

Fig. 1 | Functional diversity in DNA binding among MpARFs. a, Number of $A$. thaliana (At) and $M$. polymorpha $(M p)$ auxin response components. b,c, Ten-day-old gemmalings of Takaragaike-1 (Tak-1) wild type, Mparf1-4, pro MpARF1:MpARF1 (ARF1), pro MpARF1:MpARF2 (ARF2) and proMpARF1:MpARF3 $(A R F 3)(\mathbf{b})$ and quantification of the relative thallus areas (c). The gemmae were grown for ten days without (-) or with (+) $3 \mu \mathrm{M} 2,4-\mathrm{D}$ and normalized to control-treated Tak-1. d,e, Similarity and domain organization of MpARFs (d) and nomenclature of domain swaps (e). f, Relative thallus areas in Tak-1

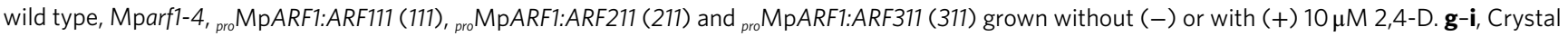
structure of MpARF2 DBD in complex with 21-7 DNA at 2.96 $\AA$ resolution (g) and structural alignment of the MpARF2 structure with MpARF1 (h) and MpARF3 (i) models. Note the lack of conserved His in MpARF3. j, PCA plot of Tak-1, Mparf1-4 and Mparf3gel-1 gemmae (inset) RNA-seq transcriptomes from three independent pools of 30-80 gemmae for each genotype. $\mathbf{k}$, Overlap of differentially expressed genes in Mparf1-4 and Mparf39el-1 gemmae. The scale bar in $\mathbf{b}$ indicates $2 \mathrm{~mm}$. The different letters in $\mathbf{c}$ and $\mathbf{f}$ indicate the significant differences tested by analysis of variance followed by Tukey's honestly significant difference $(P<0.01, n=10$ biologically independent plants). In the box plots, the centre line indicates the median, the box limits indicate the upper and lower quartiles, the whiskers indicate $1.5 \times$ the interquartile range and the circles indicate the outliers.

(Fig. 1f and Extended Data Fig. 1). Furthermore, gemma malformation was still observed, and auxin response was not restored (Fig. If and Extended Data Fig. 1c). The MpARF2 DBD is thus functionally comparable to the MpARF1 DBD, while the MpARF3 DBD is not. We solved the crystal structure at $2.96 \AA$ resolution (Fig. $1 \mathrm{~g}$ and Supplementary Table 1; Protein Data Bank (PDB) ID 6SDG) of the MpARF2 DBD in complex with a DNA oligonucleotide chosen on the basis of the Arabidopsis ARF DNA-binding preference ${ }^{12}$, and identified residues directly contacting DNA (Supplementary Fig. 1). We next generated homology models of the MpARF1 and MpARF3 DBDs on the basis of the MpARF2 DBD/DNA co-crystal structure and compared DNA-interacting interfaces. MpARF1 and MpARF2 showed identical DNA interaction interfaces, while MpARF3 deviates at a key position with Gly172 replacing a DNA-contacting histidine (Fig. 1h,i and Supplementary Fig. 1). Structural analysis thus supports the functional similarity of the MpARF1 and MpARF2 DBDs, as well as the distinction from the MpARF3 DBD.

To compare in vivo target specificity between MpARF1 and MpARF3, we performed transcriptome analysis on Mparf1 and Mparf3 mutants. Since both are important for gemma development $^{4,5,17}$, we microdissected the developing gemmae (Fig. $1 \mathrm{j}$ and Extended Data Fig. 2) and performed RNA-seq analysis. Principal 
component analysis (PCA) clustered each genotype separately, suggesting distinct gene expression profiles (Fig. 1j). Furthermore, there was limited overlap between differentially expressed genes (Fig. 1k) or over-represented Gene Ontology terms (Extended Data Fig. 3) in each mutant except for downregulated genes that may reflect impaired gemma development in both mutants. This suggests that MpARF1 and MpARF3 regulate largely different gene sets. The over-represented Gene Ontology terms did not include auxin-related functions, probably because of a scarcity of auxin-related transcriptome datasets and the absence of clear feedback regulation or highly auxin-responsive genes in $M$. poly$m o r p h a^{4}$. In bioinformatic analyses, MpARF1 and MpARF3 are coexpressed with distinct sets of transcription factors ${ }^{18}$, consistent with unique sets of target genes.

The MR defines the transcriptional activity of ARF proteins, acting as a modular activation and/or repression domain ${ }^{19}$, and MpARF1 and MpARF2 have opposing activities on a model gene when expressed in tobacco ${ }^{15}$. To investigate MR diversity, we replaced the MpARF1 MR with that of MpARF2 or MpARF3 (ARF121 and ARF131; a silent mutation was introduced in the MpARF3 MR to prevent microRNA160 regulation ${ }^{5}$ ). Neither ARF121 nor ARF131 complemented the Mparf1 mutant; instead, they induced more severe phenotypes, producing callus-like cell masses or clusters of gemma cups in both lines (Fig. 2a). Interestingly, ARF121 and ARF131 caused callus-like phenotypes in the wild-type background as well (Extended Data Fig. 4), and the phenotype resembles the effects of non-degradable Aux/IAA repressor (mIAA-GR; Fig. 2a) ${ }^{15}$, suggesting active repression. Indeed, while the auxin-activated WIP gene (Mp1g09500) was downregulated in the Mparf1 mutant, it was further repressed in the ARF121 and ARF131 plants (Fig. 2b). These results also show that auxin-activated genes are not fully repressed in the Mparf1 mutant, probably because of activation by other, auxin-independent transcription factors. Targeting active repressor domains to these loci further represses transcription. Despite the limited similarity among MRs (Fig. 1d), there is a conserved LFG motif flanked by A/B/C-class-specific residues (Fig. 2d) ${ }^{5}$. The B-ARF-specific (R/K)LFG motif was shown to recruit the TOPLESS (TPL) corepressor in various transcription factors including Arabidopsis AtARF2 ${ }^{20}$. Likewise, MpARF2, but not MpARF1, showed a clear interaction with MpTPL (Mp7g17410), which was lost when the LFG motif was substituted by alanines (Fig. 2d and Extended Data Fig. 5). A 17-amino-acid fragment encompassing the LFG motif of all MpARFs, but not LFG-to-AAA mutant versions, showed repressor activity when coexpressed with MpTPL in tobacco cells (Fig. 2e and Supplementary Fig. 2). In this assay, the 17-amino-acid fragment of MpARF1 also mediated gene repression, while the full MR activated expression in a similar assay ${ }^{15}$. Thus, regions in the MR beyond the LFG motif probably prevent MpTPL binding and gene repression. Finally, the deletion of the LFG motif in MpARF2 and MpARF3 (ARF12 1 and ARF13 ${ }^{\Delta} 1$ ) eliminated the strong phenotypes seen in the ARF121 and ARF131 lines, and partially complemented the Mparf1 mutant (Extended Data Fig. 6a), yet did not mediate auxin-dependent WIP gene activation (Extended Data Fig. 6b). We could not detect a function for the conserved LFG motif in MpARF1, as its deletion did not affect the ability to complement the Mparf1 mutant (Extended Data Fig. 6a) or to mediate auxin-dependent YUC2 (Mp8g08780) gene repression (Extended Data Fig. 6c). Thus, while MpARF1 activates transcription, MpARF2 and MpARF3 recruit MpTPL to repress transcription. We cannot, however, discard the possibility that MpARF1 functions as a repressor in a specific situation, as Arabidopsis AtARF5/MP does ${ }^{11}$.

ARF PB1 domains confer auxin dependence through interaction with Aux/IAAs (ref. ${ }^{3}$ ) and can also homo-oligomerize ${ }^{21,22}$, the in vivo relevance of which is unknown. PB1 domains were swapped among MpARFs: while ARF112 partially complemented the morphological defects of the Mparf1 mutant, ARF113 did not (Fig. 3a,b). Despite complementation under standard conditions, ARF112 did not show any response to auxin (Fig. 3a,b). MpARF2 and MpARF3 thus seem to function independently of auxin, which is consistent with the normal auxin response in Mparf3 mutants ${ }^{4,5}$ and limited Arabidopsis B/C-ARF-Aux/IAA interactions ${ }^{8,9}$. To determine the structural basis for potential differential interactions, we generated structural homology models of the MpIAA and MpARF1-3 PB1 domains. These models showed marked differences in surface charge, mainly on the negative face (Fig. 3c), which suggests unique interaction properties of each PB1 domain. In heterologous systems, the MpIAA (Mp6g05000) PB1 domain could interact with each MpARF (ref. ${ }^{15}$ ). We used docking of structural models for each protein to characterize the potential interfaces. These models suggest that both positive and negative interaction faces of the MpARF2 and MpARF3 PB1 domains engage in interaction with MpIAA, while one interaction face of MpARF1 (centred on a lysine) remains available for further interactions when in complex with MpIAA (Extended Data Fig. 7). Thus, a potential explanation for the differential auxin regulation of MpARF proteins is the ability of MpIAA to oligomerize on ARF1.

The MpARF2 PB1 domain can replace the MpARF1 PB1 domain under standard but not auxin-induced conditions (Fig. 3a,b), which suggests that the function of the MpARF2 PB1 domain is independent of auxin. MpARF1 lacking its PB1 domain (ARF110) could not complement the Mparf1 mutant (Fig. 3a,b), suggesting that homo-oligomerization is required for MpARF1 function. To test sufficiency, we replaced the MpARF1 PB1 domain with the Arabidopsis LEAFY SAM oligomerization domain (ARF11S) ${ }^{23}$. ARF11S indeed partially complemented the Mparf1 mutant defects, producing flat thalli with proper organs that were, however, auxin insensitive (Fig. 3a,b). This demonstrates the importance of homotypic interactions and suggests that this property is shared between A- and B-ARFs, but not C-ARFs, which is consistent with previous interaction assays ${ }^{15}$. Given that MpARF3 is not auxin regulated and controls different genes from MpARF1, this transcription factor seems unrelated to auxin response in $M$. polymorpha.

From these genetic and biochemical experiments, a simple model emerges: A- and B-ARFs compete for the same DNA sites, where A-ARF can switch from Aux/IAA-mediated repression to auxin-dependent activation, and B-ARF dampens auxin responsiveness through direct repression. To test whether MpARF2 indeed antagonizes MpARF1, we misexpressed a dexamethasone (DEX)-inducible MpARF2-GR protein from the constitutive

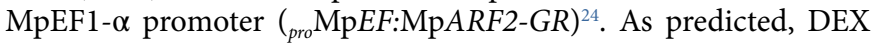
treatment conferred clear auxin resistance and rapidly repressed the expression of auxin-activated genes (Fig. 4a,b). We next increased levels of MpARF1, MpARF2 or both. MpARF1 induced growth retardation yet maintained normal auxin sensitivity, while MpARF2 overexpression caused auxin insensitivity (Extended Data Fig. 8). Joint MpARF1/MpARF2 misexpression recovered normal auxin sensitivity (Extended Data Fig. 8), which shows that MpARF2 indeed antagonizes MpARF1 and that MpARF1/2 stoichiometry, rather than absolute MpARF1 or MpARF2 levels, determines auxin responsiveness.

To determine the biological relevance of the antagonistic MpARF2 activity, we attempted to generate loss-of-function mutants through homologous recombination ${ }^{25}$ or CRISPR-Cas9 (ref. ${ }^{26}$ ) with three different single guide RNAs (sgRNAs). Neither method yielded heritable mutations despite screening 770 homologous recombination transgenics and 20 chimaeric gene-edited lines, suggesting that MpARF2 is an essential gene. We therefore generated Cre/loxP-mediated conditional knock-out sectors (Mparf2-1 $1^{\text {cko }}$ and Mparf2-2 $\left.2^{c k o}\right)^{26,27}$ through heat shock and the DEX-inducible deletion of a CRISPR-resistant MpARF2 transgene in a background with a CRISPR-Cas9-induced mutation in the endogenous 

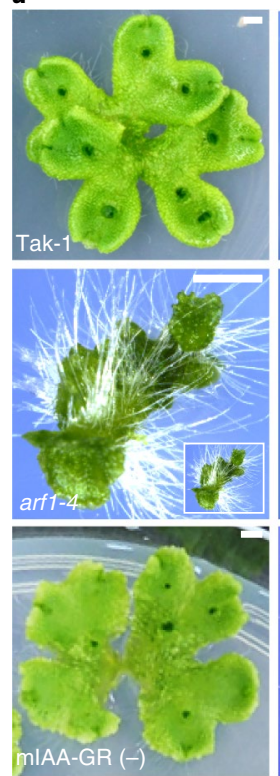
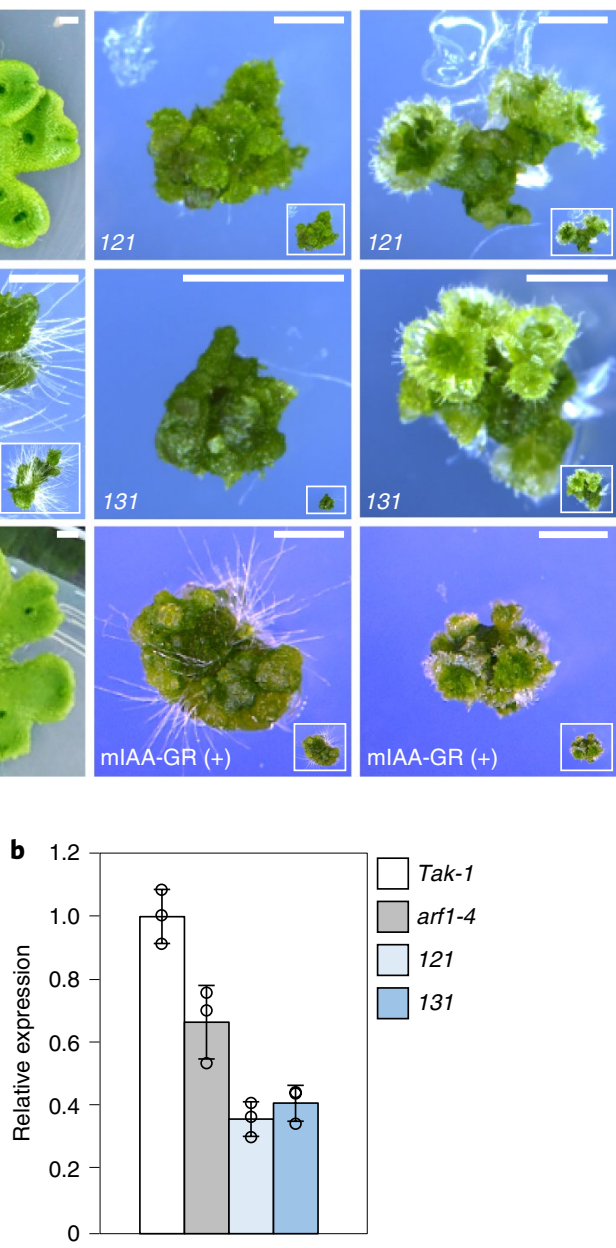

C

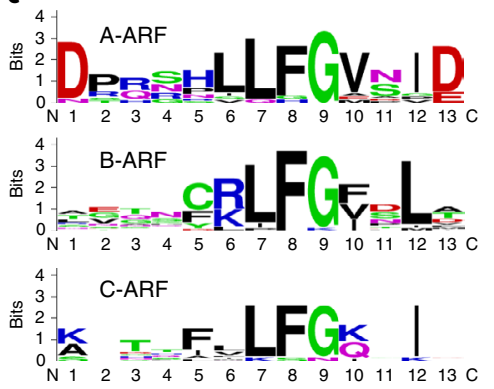

d
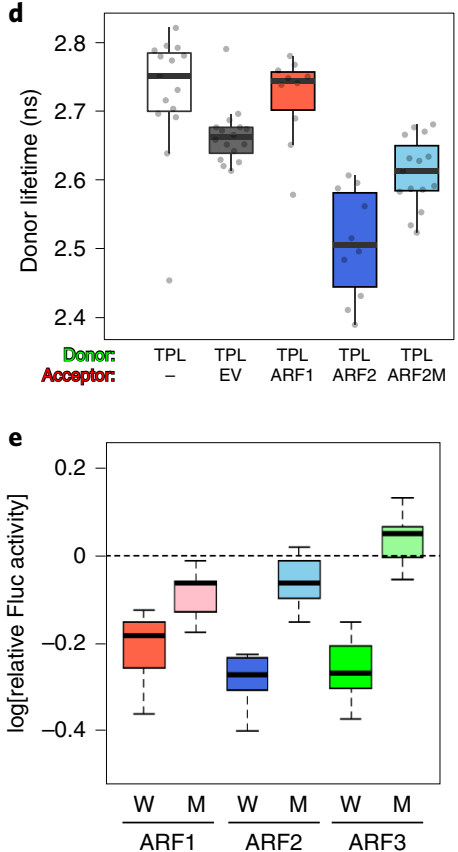

Fig. 2 | Activation and direct repression by MpARFs. a, Twenty-two-day-old gemmalings of Tak-1 wild-type, Mparf1-4, proMpARF1:ARF121 (121) and proMpARF1:ARF131 (131) lines, and 20-day-old gemmalings of mIAA-GR grown without $(-)$ or with $(+) 1 \mu \mathrm{M} D E X$. The insets show the same magnification as Tak-1. The experiment was repeated three times with similar results. b, Relative quantitative PCR (qPCR) expression of the WIP gene in Tak-1 wild type, Mparf1-4, ARF121 and ARF131. The sample consisted of three biologically independent RNA extractions from four (Tak-1) or eight (Mparf1-4, ARF121 and ARF131) plants. The error bars indicate one s.d. of the mean. The circles indicate the data points. c, Sequence logos of conserved MR motifs among Marchantia, Physcomitrella, Selaginella moellendorffii, Amborella trichopoda and Arabidopsis ARFs. d, FRET-fluorescence lifetime imaging microscopy (FLIM) interaction in Arabidopsis protoplasts: MpTPL-mNeonGreen fluorescence lifetime alone (-) or on coexpression with mScarlet-I (EV), or fusions to MpARF1, MpARF2 or MpARF2 with the LFG $\rightarrow$ AAA mutation. $n=19,15,10,10$ and 15 protoplasts (left to right). In the box plots, the centre line indicates the median, the box limits indicate the upper and lower quartiles and the whiskers indicate $1.5 \times$ the interquartile range. e, Transcriptional repression of the firefly luciferase (Fluc) transgene by coexpression of MpTPL and LFG motif peptides of MpARF1, MpARF2 or MpARF3 (W), or their LFG $\rightarrow$ AAA mutants $(M)$, relative to a control using Gal4 DBD only in tobacco leaves. $n=9$ biologically independent samples. The scale bars in a indicate $2 \mathrm{~mm}$.

MpARF2 gene (Extended Data Fig. 9a,b). The elimination of the additional $\mathrm{Mp} A R F 2$ copy triggered nuclear-localized tdTomato expression, leading to fluorescent mutant sectors (Extended Data Fig. 9c,d). Without induction, Mparf $2^{\text {cko }}$ mutants produced normal thalli (Fig. 4c and Extended Data Fig. 9c), but Cre induction caused growth retardation and abnormal morphology in young gemmalings (Fig. 4c and Extended Data Fig. 9c). When Cre was induced in mature thalli, severe growth arrest occurred at the thallus tips, while the gemma cups kept growing (Fig. 4d). These results suggest that antagonistic MpARF2 activity to MpARF1 is critical for the development of the M. polymorpha thallus.

Antagonism by direct competition between MpARF1 and MpARF2 is realistic only if their DNA-binding affinities for the same DNA target are within the same range, or when there are large differences in protein concentrations. We therefore measured the binding affinities of recombinant MpARF1 and MpARF2 DBDs to the same
DNA site using a single-molecule fluorescence resonance energy transfer (FRET)-based assay (Fig. 4e) ${ }^{28}$. The proteins were titrated on immobilized DNA oligonucleotide labelled with FRET-compatible fluorophores (Extended Data Fig. 10a), such that protein binding would alter FRET efficiency (Extended Data Fig. 10b), measured at a single-molecule resolution (Extended Data Fig. 10c). As DNA, we used the ER7 sequence consisting of two canonical ARF binding sites spaced by seven nucleotides ${ }^{12}$ (Extended Data Fig. 10a), similar to the one co-crystallized with the MpARF2 DBD (Fig. 1g) and that should bind to both proteins on the basis of the homology models (Fig. 1h). Using this assay, we derived a dissociation constant of $12 \mathrm{nM}$ for the MpARF1 DBD and $61 \mathrm{nM}$ for the MpARF2 DBD (Fig. 4f), well within one order of magnitude, and compatible with competition at near-stoichiometric protein concentrations.

Finally, to define the true endogenous protein levels and accumulation patterns of MpARF1 and MpARF2, we generated genomic 
a
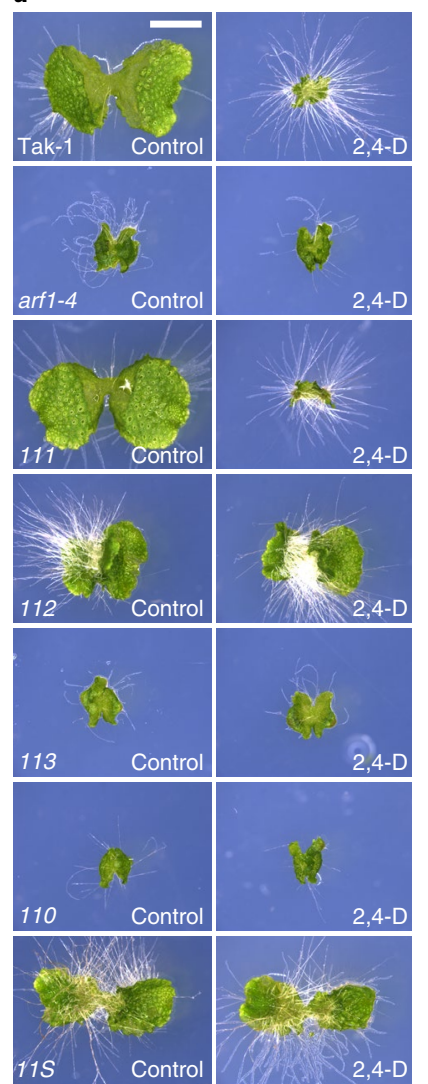
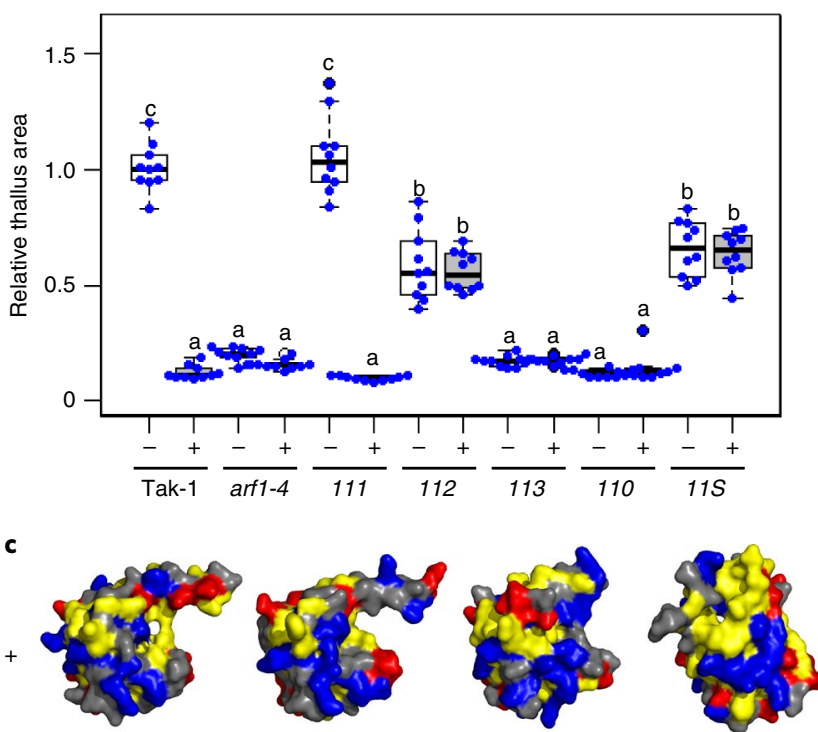

$\stackrel{180}{\circ}^{\circ} \mathrm{ARF} 1$

ARF2

ARF3

IAA
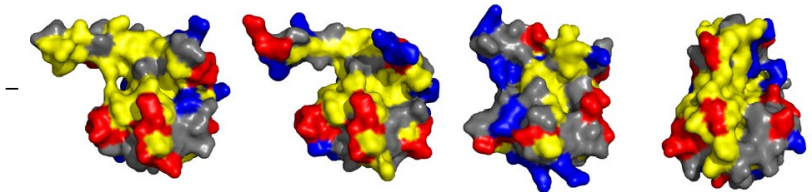

Fig. 3 | Dual function of the PB1 domain. a, Ten-day-old gemmalings of Tak-1 wild-type, Mparf1-4, pro MpARF1:ARF111 (111), pro MpARF1:ARF112 (112),

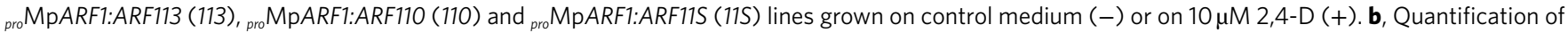
the thallus areas in a relative to Tak-1. Different letters indicate significant differences tested by analysis of variance followed by Tukey's honestly significant difference ( $P<0.01, n=10$ biologically independent plants). In the box plots, the centre line indicates the median, the box limits indicate the upper and lower quartiles and the whiskers indicate $1.5 \times$ the interquartile range. The blue dots indicate the data points. The experiment was repeated five times with similar results. c, Charge distribution (blue is positive, red is negative and yellow is hydrophobic) on surface representations of the MpARF1, MpARF2, MpARF3 and MpIAA PB1 domains. The top row shows the positive (K) side, and the bottom row shows the negative (OPCA) side. The scale bar in a indicates $2 \mathrm{~mm}$.

knock-in carboxy-terminal translational fusions to mScarlet-I fluorescent protein. Given that the loss and gain of function of both MpARF1 and MpARF2 cause strong developmental defects (Fig. 1b, Fig. 4 c,d, Extended Data Figs. 8 and 9, and ref. ${ }^{12}$ ), the normal phenotype of these knock-in lines (Fig. $4 \mathrm{~g}, \mathrm{~h}$ ) demonstrates that these lines report endogenous protein levels. While MpARF1-mScarlet-I broadly accumulates in gemmae (Fig. 4g), MpARF2-mScarlet-I is restricted to regions surrounding the apical notch (Fig. 4h). Importantly, the spatial distribution patterns of both proteins define distinct zones of cells that express only MpARF1 and MpARF2 in different stoichiometries, and should thus confer differential auxin sensitivity.

Our study reveals a simple model revolving around two competing transcription factors underlying auxin response in the minimal M. polymorpha system (Fig. 4i): A-ARF is the only auxin-sensitive transcriptional regulator and can switch from Aux/IAA-mediated repression to activation in an auxin-dependent manner. B-ARF functions independently of auxin and antagonizes A-ARF by competing for target sites and by recruiting the TPL corepressor. The expression patterns of A-ARF and B-ARF thus create zones with different auxin sensitivities, in addition to the MpIAA expression level. It is intuitive how auxin response may have evolved by influencing the stoichiometry of a pre-existing antagonistic ARF pair ${ }^{29}$. This basic module may form the basis for the auxin response diversity in species with expanded gene families. The duplication of A- or $\mathrm{B}-\mathrm{ARF}$ s would change A/B stoichiometry and allow duplicated genes to escape ancestral regulation (for example, by the gain of Aux/IAA interaction in B-ARFs) $)^{8,9}$. Strikingly, this simple model is consistent with most, if not all, studies in the more complex Physcomitrella and Arabidopsis systems, rationalizing genetic interactions $^{10}$, protein interactions ${ }^{8,9}$, activity assays $s^{7,11}$ and $A R F$ gene expression pattern $s^{30}$, and may therefore represent a universal unit at the base of the complex auxin response networks.

\section{Methods}

Plant materials and growth conditions. The male $M$. polymorpha strain Tak-1 was used as the wild type. The female strain Tak-2 was used to generate spores for transformation. The methods of cultivating and transforming $M$. polymorpha were described previously ${ }^{31-33}$. Nicotiana tabacum was used in the transcriptional repression assays. The A. thaliana strain Col-0 was used to generate protoplasts for the FRET-FLIM experiments.

Promoter and domain swap experiments. The promoter region of MpARF1 (including three kilobases $(\mathrm{kb})$ upstream from the transcriptional start site) was amplified with the primer set HK120/HK125 (see Supplementary Table 2 for all oligonucleotide sequences) and cloned into pMpGWB301 (Addgene) using the XbaI site (pHKDW046). For the promoter swap experiments, the genomic coding sequences (CDS) of MpARFs were amplified with the following primer sets: MpARF1, HK009/HK015; MpARF2, HK108/HK119; MpARF3, HK033/ HK097. These sequences were subcloned into pENTR/D-TOPO (Thermo Fisher 

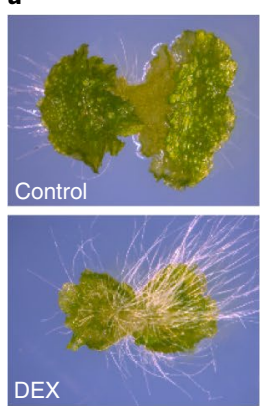

b
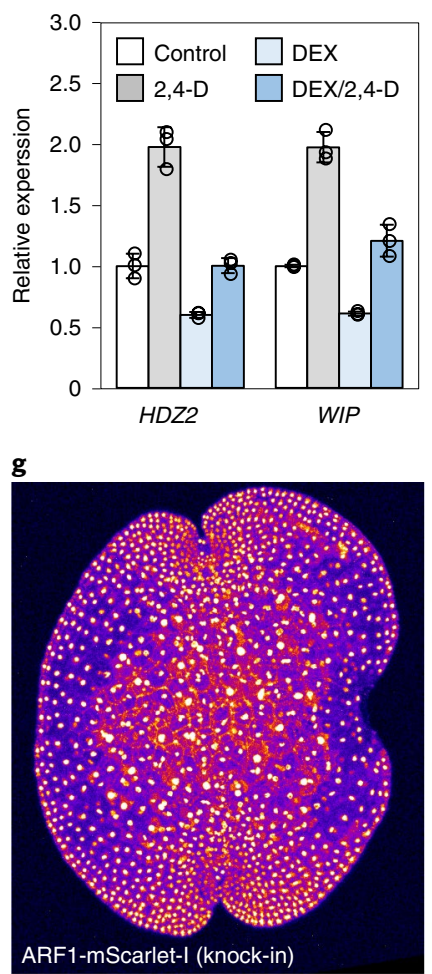

Min
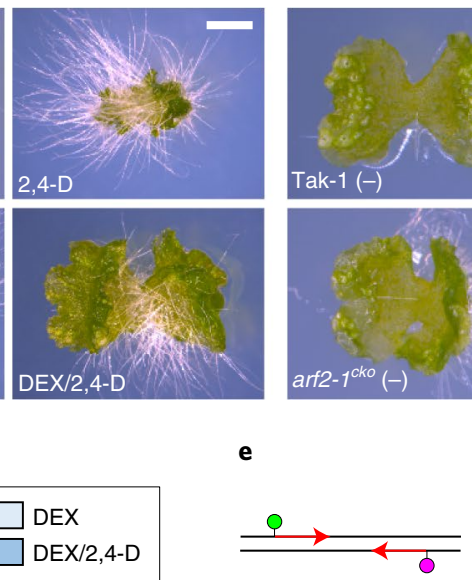

hi-FRET

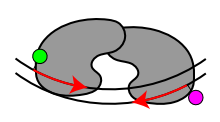

Io-FRET d
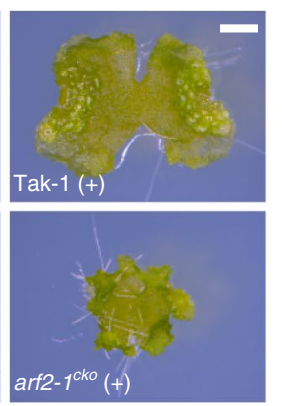
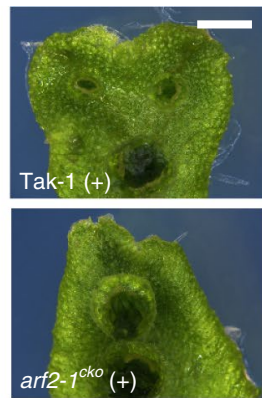

f

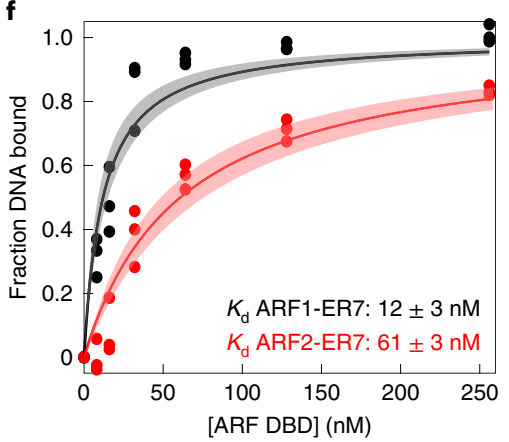

i

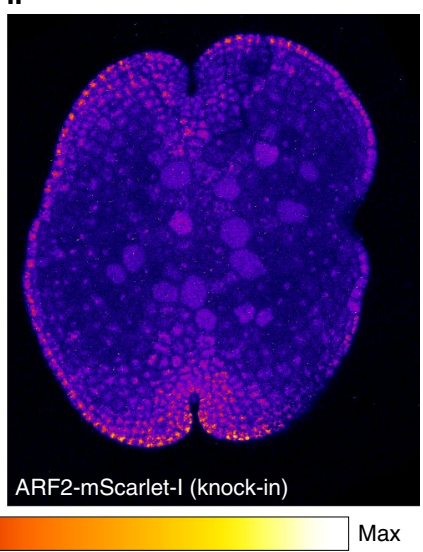

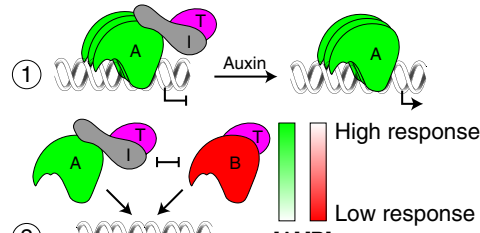

(2) $00000000 \quad[\mathrm{~A}][\mathrm{B}]$
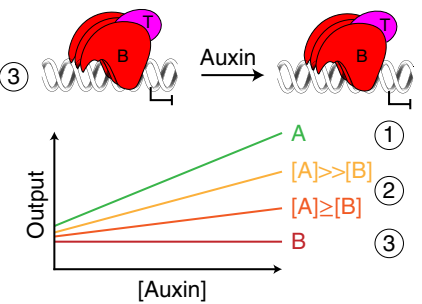

Fig. 4 | A/B-ARF antagonism defines a minimal auxin response model. a, Ten-day-old gemmalings of a proF:MpARF2-GR line grown on control medium, on $3 \mu \mathrm{M} 2,4-\mathrm{D}$, on $1 \mu \mathrm{M}$ DEX or both. The experiment was repeated two times with similar results. $\mathbf{b}$, Relative qPCR expression of HDZ2 (also known as C2HDZ; Mp2g24200) and WIP genes in a preF:MpARF2-GR line treated with control medium, with 10 $\mu \mathrm{M} 2,4-\mathrm{D}$, with $10 \mu \mathrm{M}$ DEX or both, for $1 \mathrm{~h}$. The sample consisted of three biologically independent RNA extractions from four plants. The error bars indicate one s.d. of the mean. The circles indicate the data points. c,d, Eight-day-old gemmalings (c) and two-week-old thallus tips (d) of Tak-1 or Mparf2-7cko grown on control medium without heat shock $(-)$ or with $1 \mu \mathrm{M}$ DEX and a heat shock (+) on day 1. The experiment was repeated four times with similar results. e, FRET-based assay for measuring ARF binding to inverted repeat ARF binding sites (red arrows). On protein binding, the FRET efficiency between dyes (magenta and green) changes. $\mathbf{f}$, Quantification of protein-DNA binding, expressed as the fraction of bound DNA molecules at varying MpARF1 and MpARF2 DBD concentrations. The lines show the fit through the raw data within 95\% confidence intervals. Square brackets indicate concentration. $n=3$ independent measurements for each protein concentration. $K_{\mathrm{d}}$, apparent protein-DNA dissociation constant. $\mathbf{g}, \mathbf{h}$, Accumulation of MpARF1-mScarlet-I (g) and MpARF2-mScarlet-I (h) fusion proteins in genomic knock-in lines in mature gemmae on a false-colour intensity scale. The experiment was repeated five times ( $>25$ gemmae) with similar results. i, Minimal auxin response model: (1) at low auxin concentrations, A-ARF (A) recruits Aux/IAA (I) and TPL (T) to repress genes; at high auxin concentrations, I is degraded and A activates genes. (2) A and B-ARF (B) compete for binding to the same targets, where the stoichiometry of both determines the auxin responsiveness. (3) B recruits $T$ to repress targets independently of auxin. The graph shows that the presence and stoichiometry of $A$ and $B$ define the cellular responsiveness to auxin. The scale bars indicate $1 \mathrm{~mm}$ in $\mathbf{a}$ and $\mathbf{c}$ and $2 \mathrm{~mm}$ in $\mathbf{d}$.

Scientific). The genomic CDS cassettes were then transferred into pHKDW046 using the Gateway LR Clonase II Enzyme mix (Thermo Fisher Scientific). The DBDs of MpARFs were amplified and attached with PacI and PmeI restriction enzyme sites by PCR using the following primer sets: MpARF1, HK009/HK011; MpARF2, HK018/HK020; MpARF3, HK112/HK113. They were then subcloned into pENTR/D-TOPO. The MRs of MpARFs with the PacI site at the $5^{\prime}$ end and the PmeI site at the $3^{\prime}$ end were amplified from complementary DNA using the following primer sets: MpARF1, HK116/HK120; MpARF1 with stop, HK116/ HK285; MpARF2, HK021/HK022; MpARF3, HK030/HK031. The PB1 domains of MpARFs and the SAM domain of AtLFY with the PmeI site at the $5^{\prime}$ end and the AscI site at the $3^{\prime}$ end were amplified from CDNA of $M$. polymorpha or A. thaliana using the following primer sets: MpARF1, HK014/HK015; MpARF2, 
HK023/HK024; MpARF3, HK032/HK033; SAM, HK481/HK482. The amplified MRs, PB1s and SAM were then fused with the DBDs in various combinations using the restriction enzyme sites PacI, PmeI and AscI. The resultant chimaeric CDS were transferred into pHKDW046 with the LR reaction to fuse with the MpARF1 promoter. At least three independent transformants showing similar phenotypes were analysed for each construct.

Recombinant protein expression and purification. The DBDs for MpARF1 and MpARF2 used in the single-molecule DNA-binding assays and crystallography were amplified using oligonucleotides MpARF1-DBD-FW/ MpARF1-DBD-RV (MpARF1) and MpARF2-DBD-FW/ MpARF2-DBD-RV (MpARF2), and cloned into a modified pTWIN1 vector ${ }^{12}$ by SLiCE cloning ${ }^{34}$. Expression and purification were performed as previously described for the AtARF1 and AtARF5 DBDs ${ }^{12}$.

\section{Crystal structural analysis. The oligonucleotide}

$5^{\prime}$-d(TTGTCGGCGATTCGCCGACAA)-3' was annealed with an equimolar amount of the complementary strand, both purchased from Biomers. MpARF2-DBD (Uniprot A0A0K2QVG1; residues 1-395) was purified using a procedure equivalent to that used for AtARF1-DBD (ref. ${ }^{12}$ ).

The MpARF2:DNA complex was prepared by diluting the required amount of double-stranded DNA (dsDNA) in a solution of $20 \mathrm{mM}$ Tris $(\mathrm{pH}=8.0)$, $500 \mathrm{mM} \mathrm{NaCl}$ and $20 \mathrm{mM}$ dithiothreitol and by subsequently adding this solution to a solution of MpARF2 in the same buffer, to a final 2:1 (protein:dsDNA) stoichiometry and a final MpARF2 concentration of $5 \mathrm{mg} \mathrm{ml}^{-1}$. The complex thus obtained was crystallized by applying the sitting-drop vapor-diffusion method using $0.2 \mathrm{M}$ sodium formate and $20 \% \mathrm{w} / \mathrm{v}$ polyethylene glycol 3,350 as the crystallization buffer at $18^{\circ} \mathrm{C}$, in drops of $1 \mu \mathrm{l}+1 \mu \mathrm{l}$ (complex:reservoir).

Cube-shaped crystals appeared in less than a week and were frozen within a day of appearance. Cryo-cooling in liquid nitrogen was carried out using a cryo-protecting solution containing reservoir solution supplemented with $10 \%$ glycerol. The X-ray diffraction experiments were performed at beamline BL13 XALOC (ref. ${ }^{35}$ ) (ALBA Synchrotron), using a wavelength of $0.9792 \AA$ at $100 \mathrm{~K}$. The data were processed using the Global Phasing AutoPROC program ${ }^{36}$ applying anisotropic cutoffs ${ }^{37}$

The structure was solved with Phaser $^{38}$ using the dimerization domain of AtARF1-DBD (PDB ID 4LDX). A near-complete initial model was obtained with Phenix autobuild ${ }^{39}$. The structure was completed through alternate manual mode building with Coot v.0.8.9 (ref. ${ }^{40}$ ) and refinement with PHENIX v.1.16_3549 (ref. ${ }^{39}$ ). The Ramachandran statistics of the final refined model were $93.37 \%$ favoured, $5.83 \%$ allowed and $0.81 \%$ outliers. The final model validation parameters were: Clashscore of 16.74 , root mean square deviation (RMS) (bonds) of $0.013 \AA$ and RMS (angles) of $1.46^{\circ}$. The crystallographic parameters are given in Supplementary Table 1. The figures were prepared using PyMOL v.2.4.0a0 (www.pymol.org; Schrödinger LLC)

Homology modelling and docking. The crystal structure of MpARF2 (PDB ID 6SDG) was used as template for homology modelling on Swissmodel (https:// swissmodel.expasy.org/). The MpARF1 and MpARF3 protein sequences and the MpARF2 template were uploaded to the Swissmodel server, and the modelling was run with the default configuration. The global and per-residue model qualities were ranked using the QMEAN scoring function ( -4.86 for the MpARF1 model and -5.92 for the MpARF3 model).

The homology models of the PB1 domain were generated using Modeller v.9.17 (ref. ${ }^{41}$ ) (for MpARF1, MpARF2 and MpIAA) and the Phyre2 web server ${ }^{42}$ (for MpARF3). The structures of the PB1 domains of AtARF5 (PDB ID 4CHK) and AtIAA17 (PDB ID 2MUK) were used as templates for modelling Marchantia ARFs and IAA, respectively. In Modeller, 100 random models were generated, where the model with lowest discrete optimized protein energy score was chosen as the best model. In Phyre2, the modelling was performed under normal mode. The obtained homology models were visualized using PyMOL v.2.2 (www.pymol.org; Schrödinger LLC). The surface representations of various residues are coloured according to their charges and hydrophobicity. Hydrophobic residues (Ala, Gly, Val, Ile, Leu, Phe, Met and Pro) are coloured with yellow, polar residues (Asn, Gln, Thr, Ser, Cys, Tyr and Trp) are coloured with grey, positively charged residues (Arg, Lys and His) are coloured with blue and negatively charged residues (Glu and Asp) are coloured with red.

For protein-protein docking, we used the HADDOCK2 web server ${ }^{43}$ in the easy interface mode. The modelled $\mathrm{PB} 1$ domains were used for docking, where both the positive $(\mathrm{K})$ and negative (OPCA) face residues were provided as the active site residues. The cluster with the lowest HADDOCK score and $z$-score was considered as the best docked pose for that pair. The obtained poses were visualized in PyMOL (www.pymol.org; Schrödinger LLC), and the residues in dimer interface were calculated using a Python script (http://www.protein.osaka-u. ac.jp/rcsfp/supracryst/suzuki/jpxtal/Katsutani/InterfaceResidues.py).

Modified pseudo-Schiff propidium iodide staining for developing gemmae. The gemma cups were transversely sectioned into two or three pieces by knife. The slices of the gemma cups were stained by pseudo-Schiff propidium iodide staining ${ }^{44}$ with some modifications. The tissues were incubated in a fixative solution ( $75 \%$ ethanol, $25 \%$ acetic acid) at $4{ }^{\circ} \mathrm{C}$ for $3 \mathrm{~d}$ followed by washing with $80 \%$ ethanol. The tissues were then treated with $1 \%$ periodic acid for $1 \mathrm{~h}$ and stained with Schiff reagent containing $0.1 \mathrm{mg} \mathrm{ml}^{-1}$ propidium iodide for $1 \mathrm{~h}$. Confocal laser scanning microscopy was performed using a Leica TCS-SP5 microscope. The excitation wavelength was $561 \mathrm{~nm}$, and the detection range was set from 600 to $700 \mathrm{~nm}$. Z-stack images were obtained with $0.5 \mu \mathrm{m}$ thickness. The optical section images were generated using Volume Viewer in Fiji software ${ }^{45}$.

RNA-seq analysis using developing gemmae. To isolate developing gemmae, thallus tips were grown for one week, and the youngest gemma cups close to the meristematic notches were collected. Gemma isolation was performed following the protocol for isolating Arabidopsis embryos from ovules with some modifications ${ }^{46}$. A CellTrics $50 \mu \mathrm{m}$ filter (Sysmex) was used to remove debris from the crushed gemma cup mixture. For each sample, 30 to 80 developing gemmae were isolated by micromanipulator. The RNA was extracted using TRIzol Reagent (Thermo Fisher Scientific) with the following modifications. Homogenization was performed at $60^{\circ} \mathrm{C}$ for $30 \mathrm{~min}$. After we added isopropanol, the samples were mixed with $1.5 \mu \mathrm{l}$ of GlycoBlue Coprecipitant (Thermo Fisher Scientific) and incubated at $-20^{\circ} \mathrm{C}$ overnight. The extracted RNA was treated with an RNase-free DNase set (QIAGEN), and purified with an RNeasy Minelute Cleanup Kit (QIAGEN). The reverse transcription and cDNA amplification were performed following the published protocol ${ }^{47}$. The DNA library for sequencing was prepared with a SMARTer ThruPLEX DNA-Seq Kit (Takara Bio) and sequenced with NovaSeq6000 (paired-end 150 base pairs).

The quality assessment for the raw RNA-seq reads was performed using FastQC (www.bioinformatics.babraham.ac.uk/projects/fastqc). The Illumina adapters at the ends of the paired reads were cleaned up using TrimGalore (v.0.5.0; https://github.com/FelixKrueger/TrimGalore) with the following parameters: -stringency 5; -paired -length 100; -clip_R1 30;-clip_R2 30. The cleaned FASTQ reads were mapped onto the $M$. polymorpha genome (v.3.1 accessed through Phytozome) using HISAT2 v.2.1.0 (ref. ${ }^{48}$ ) with the default parameters with -dta as an additional setting. The post-processing of the SAM/BAM files was performed using SAMTOOLS v.1.9 (ref. ${ }^{49}$ ). FeatureCounts v.1.6.2 (ref. ${ }^{50}$ ) was used to count the raw reads corresponding to each gene, with the parameters $-t$ exon -g gene_name -Q 30 -p -primary. DEseq2 (ref. ${ }^{51}$ ) was used to normalize the raw counts and perform the differential expression analysis $\left(P_{\mathrm{adj}}<0.05\right)$. All the sequenced raw reads were deposited in the NCBI Sequence Read Archive (SRA) under the project accession number PRJNA554398 (http://www.ncbi.nlm.nih.gov/ bioproject/554398).

Gene Ontology analysis. The Gene Ontology enrichment analysis was performed using the PlantRegMap server (http://plantregmap.cbi.pku.edu.cn/go.php). All genes in $M$. polymorpha were used as the background model, and the significantly over-represented categories were tested using Fisher's exact test, with a $P$ value cut-off of 0.01

Quantitative PCR with reverse transcription. To measure the transgene expression levels in the promoter-swap lines, RNA was isolated from the gemmalings, and cDNA was synthesized from $0.5 \mathrm{mg}$ of total RNA with the ReverTra Ace qPCR RT Master Mix with gDNA Remover (TOYOBO). Quantitative PCR with reverse transcription (qRT-PCR) was performed using the KOD SYBR qPCR Mix (TOYOBO) and a LightCycler 96 (Roche). The primer set (HK618 and HK619) was used to amplify the transgene-specific $5^{\prime}$ untranslated region. A three-step cycle consisting of denaturation at $98^{\circ} \mathrm{C}$ for $10 \mathrm{~s}$, hybridization at $60^{\circ} \mathrm{C}$ for $5 \mathrm{~s}$ and elongation at $68^{\circ} \mathrm{C}$ for $30 \mathrm{~s}$ was repeated 40 times and then followed by a dissociation step. The transcript levels were normalized to the EF1- $\alpha$ gene.

To test auxin-dependent gene expression, the auxin treatment on ten-day-old gemmalings, RNA extraction, cDNA synthesis and qRT-PCR were performed as previously described ${ }^{4}$. The DEX treatment was performed similarly to the auxin treatment.

FRET-FLIM. For FRET-FLIM, fusions of MpTPL were made in pMON999-mNeonGreen, and fusions of MpARF1, MpARF2 and the LFG-AAA mutant of MpARF2 were made in pMON999-mScarlet-I vectors using the primers listed in Supplementary Table 2 and using SLiCE cloning ${ }^{34}$.

The fluorescent proteins were coexpressed in Arabidopsis leaf mesophyll protoplasts, and the FLIM analysis was performed as described previously ${ }^{52}$. The measurements were conducted on a Leica TCS SP8 X system equipped with hybrid detectors and a pulsed white-light laser. The system was used for the detection of mNeonGreen and mScarlet-I, which were excited at $488 \mathrm{~nm}$ and $561 \mathrm{~nm}$, and detected between $520-551 \mathrm{~nm}$ and 585-607 nm, respectively. From the fluorescence intensity images, the decay curves were calculated per pixel and were fitted with a double-exponential decay model using SPCImage v.7.1 (Becker \& Hickl). For all samples, a double-exponential model function was used without fixing any parameters.

Transcriptional repression assay. The GAL4 DBD sequence was amplified with the primer set GAL4BD_RwithXbaI/ GAL4BD_FwithXbaI and cloned 
into the XbaI site of pMpGWB102 (Addgene) to generate pMpGWB102GAL4DBD that contains a cassette for cauliflower mosaic virus $35 \mathrm{~S}$ promoter:GAL4DBD-GATEWAY:Nos terminator. The LFG motif sequences were amplified with partially overlapping primer pairs (Supplementary Table 2) and subcloned into the pENTR/D-TOPO. The generated LFG motifs were transferred into pMpGWB102-GAL4DBD using the LR Clonase II enzyme mix. MpTPL cDNA was amplified from Tak-1 thallus cDNA with the primer set MpTPL_F/MpTPL_R1, subcloned into the pENTR/D-TOPO and transferred into the pMpGWB102 using the LR Clonase II enzyme mix. The Renilla luciferase (Rluc) coding sequence and the reporter construct (35S enhancer-6xUAS$35 \mathrm{~S}$ minimal promoter:firefly luciferase) were amplified with the primer sets RenillaLUCforTOPOF/RenillaLUCstopR and 6xUASfLUCcloningFforTOPO/6xU ASfLUC_stopR, respectively, subcloned into the pENTR/D-TOPO and transferred into pMpGWB102 and pMpGWB101 using the LR Clonase II enzyme mix. The plasmids used as templates for the amplification of Gal4 DBD, Rluc and the reporter construct were described previously ${ }^{53}$.

N. tabacum plants were grown under continuous white light at $22^{\circ} \mathrm{C}$ for four to five weeks. Agrobacterium harbouring effector, coeffector, reporter or internal control vectors were cultured in liquid LB media containing selection antibiotics at $28^{\circ} \mathrm{C}$. Mixtures of the four cultures (26:15:30:4) were centrifugated at 2,600 $\mathrm{g}$ for $5 \mathrm{~min}$, resuspended in MES buffer $\left(10 \mathrm{mM} \mathrm{MgCl}_{2}, 10 \mathrm{mM}\right.$ MES and $200 \mu \mathrm{M}$ acetosyringone) to an OD600 of 1.0 and incubated at $28^{\circ} \mathrm{C}$ for $4 \mathrm{~h}$ before infiltration. Infiltration was performed according to the methods described previously $^{54}$. A set of the Agrobacterium mixtures was infiltrated at eight to nine points, and the transient expression was assayed $3 \mathrm{~d}$ after inoculation. Fluc and Rluc activities were assayed using the dual-luciferase assay reagents (Promega) and the microplate reader POWERSCAN4 (DS PHARMA BIOMEDICAL). Three days after the inoculation, a leaf disc of approximately $5 \mathrm{~mm} \times 5 \mathrm{~mm}$ was removed and ground in $200 \mu \mathrm{l}$ of passive lysis buffer. Then, $20 \mu \mathrm{l}$ of lysate supernatant was incubated with $100 \mu \mathrm{l}$ of the Luciferase Assay Reagent, and the chemiluminescence was measured. After the first measurement, $100 \mu$ of the Stop \& Glo Reagent was added, and a second measurement was taken. The relative luciferase activity of each effector condition was calculated as the ratio between the Fluc and the Rluc (standard) activity and divided by that of the control effector condition (Gal4 DBD without the LFG motif) from the same leaf to normalize differences among leaves.

Overexpression of MpARF1 and MpARF2. To generate ${ }_{p r o} E F: M p A R F 2-G R$, the genomic CDS of MpARF2 were amplified with the primer set HK108/HK109, subcloned in the pENTR/D-TOPO vector and then transferred into pMpGWB113 to insert between the MpEF1- $\alpha$ promoter and GR $\left(\right.$ ref. ${ }^{20}$ ). To generate

pro EF:MpARF1, MpARF1 cDNA was amplified by RT-PCR using mRNA from Tak-1 as the template with the primer set ARF1CDS_inf_F1/ARF1CDS_inf_R1, cloned into the pENTR-1A vector using an In-Fusion HD Cloning Kit (TaKaRa) and then transferred to pMpGWB303. To generate ${ }_{p r o} E F: M p A R F 2$, MpARF2 cDNA was amplified as for MpARF1 with the primer set MpARF2_clf_TOPO/MpARF2_stop, cloned into the $\mathrm{pENTR/D-TOPO}$ vector and then transferred to $\mathrm{pMpGWB103.}$

Accessible volume simulations. The web server 3D-DART (ref. ${ }^{55}$ ) was used to model a standard B-DNA structure for the oligo used in the single-molecule FRET (smFRET) experiments and to bend it using the geometrical information extracted from the short DNA present in the crystal structure of MpARF2. The standard B-DNA oligo and the bent oligo bound to MpARF2 were then used as starting point to model the accessible volumes of the FRET pair. The accessible volumes were calculated with FRET-restrained positioning and screening software ${ }^{56}$ using the geometric characteristics for the dyes reported previously $y^{57}$.

Single-molecule measurement of the dissociation constant. The imaging was performed on a home-built TIRF microscope as previously described ${ }^{58}$. The measurements were performed using alternating-laser excitation; in this excitation scheme, a frame where the donor is excited is alternated with a frame where the acceptor is excited. The emission of the fluorophores is spectrally divided into two different channels on the emCCD camera sensor. This creates three photon streams: donor emission after donor excitation (DD), acceptor emission after donor excitation (DA, arising from FRET) and acceptor emission after acceptor excitation (AA). These photon streams can be used to calculate the raw FRET efficiency $\left(E^{*}=\mathrm{DA} /(\mathrm{DD}+\mathrm{DA})\right)$ and stoichiometry $(S=(\mathrm{DD}+\mathrm{DA}) /$ $(\mathrm{DD}+\mathrm{DA}+\mathrm{AA})) \cdot E^{\star}$ contains the information about the relative distance of the two fluorophores, whereas $S$ contains the information about the labelling state of a given molecule (allowing us to filter out molecules missing an active donor or an active acceptor)

The camera acquisition time and the excitation time were set to $250 \mathrm{~ms}$; the laser powers were set to $0.5 \mathrm{~mW}$ for both green $(\lambda=561 \mathrm{~nm})$ and red $(\lambda=638 \mathrm{~nm})$ lasers. The imaging buffer contained $137 \mathrm{mM} \mathrm{NaCl}, 2.7 \mathrm{mM} \mathrm{KCl}$, $10 \mathrm{mM}$ phosphate, $1 \mathrm{mM}$ Trolox, $1 \%$ gloxy and $1 \%$ glucose. Trolox is a triplet state quencher ${ }^{59}$; gloxy is an enzymatic system that uses glucose as a substrate for oxygen removal, decreasing fluorophore bleaching ${ }^{60}$.

Single-molecule titrations. The labelled dsDNA oligos were immobilized on a PEGylated glass coverslip as described previously ${ }^{61}$. The PEGylation was carried out inside the wells of a silicone gasket placed on the coverslip (Grace Bio-labs). Each titration was performed using a single well, washing it between data points with $\sim 600 \mu \mathrm{l}$ of PBS $1 \mathrm{X}$. The final washing step consisted of three washings separated by $15 \mathrm{~min}$. Each data point consisted of three movies (1,000 frames each); to allow the system to equilibrate, a waiting step of $5 \mathrm{~min}$ was added before starting the acquisition of the first movie.

Mutagenesis of MpARF2. For the mutagenesis of MpARF2 by homologous recombination, homologous arms were amplified from MpARF2 genomic DNA by using the primer set MpARF2-gt_F1/ MpARF2-gt_R1 and MpARF2-gt_F2/ MpARF2-gt_R2 and cloned into pJHY-TMp1 (ref. ${ }^{21}$ ). The resultant plasmid was transformed into F1 spores generated by crossing Tak-1 to Tak-2 through the Agrobacterium-mediated method ${ }^{28}$. To check for recombination, genomic PCR was performed using the primer sets MpARF2-L2/MpEFp_GT_R1, MpARF2-L6/ MpARF2-R12 and MpARF2-R1/HIF.

For the mutagenesis of MpARF2 by the CRISPR-Cas9 method, sets of two oligo DNAs (sgRNA1: HK184/HK185, sgRNA2: HK186/HK187, sgRNA3: HK188/ HK189) were annealed and cloned in pMpGW_En03 (ref. ${ }^{22}$ ). The resultant cassettes expressing sgRNA and Cas9 were transferred into pMpGE010 by the LR reaction. To generate sgRNA-resistant MpARF2, the cDNA sequence including CDS was amplified with the primer set HK119/HK282 and cloned into the pENTR/D-TOPO vector. The target site of sgRNA1 was mutated by PCR with the primer set HK279/HK280 and self-ligation. The MpARF2 promoter region including $3 \mathrm{~kb}$ upstream of the transcriptional start site was also amplified with the primer set HK107/HK281 and cloned into the pENTR/D-TOPO vector. The MpARF2 promoter and sgRNA-resistant CDS were combined using the EcoRI and $A s c \mathrm{I}$ sites. The resultant cassette was transferred into pMpGWB337 by the LR reaction ${ }^{23}$. To check the mutation in the MpARF2 locus, genomic PCR was performed with the primer set HK283/HK284, which binds introns and thus amplifies only endogenous MpARF2 copy. To delete the sgRNA-resistant MpARF2 cassette from the mutant genome, mutants were planted on the medium with $1 \mu \mathrm{M}$ DEX, incubated at $37^{\circ} \mathrm{C}$ for $1 \mathrm{~h}$ after $1 \mathrm{~d}$ of cultivation and then placed back under the normal growth conditions. The primer set HK064/HK190 was used to check the deletion of ARF2m by genomic PCR.

Genomic knock-in translational fusions for MpARF1 and MpARF2. Two homologous arms of $\sim 3.6 \mathrm{~kb}$ each were amplified by PCR from genomic DNA. The 3' end of the genomic CDS excluding the stop codon was amplified using the oligonucleotides SJD005/SJD006 (MpARF1) or SJD009/SJD010 (MpARF2), and another arm from the non-coding region after the stop codon was amplified using SJD007/SJD008 (MpARF1) or SJD011/SJD012 (MpARF2). These fragments were cloned into the pJHY-TMp1 vector ${ }^{21}$ at the HindIII and AscI sites, respectively, using the SLiCE cloning method ${ }^{34}$. The fluorescent protein $\mathrm{mScarlet}-\mathrm{I}$ was amplified using the oligonucleotides SJD003/SJD004 and cloned downstream of the first homologous arm to be translationally fused with MpARF1/2. The transformation into spores was performed as previously described ${ }^{28}$. The successfully knocked-in lines were screened from 6 (MpARF1) and 78 (MpARF2) hygromycin-resistant transformants by genomic PCR using the primer sets HK056/SJD091 and HK050/SJD149 for MpARF1 and HK072/SJD094 and HK063/ HK149 for MpARF2.

The live cell confocal imaging was performed on mature gemmae using a Leica SP8X-SMD confocal microscope equipped with a hybrid detector and a pulsed white-light laser. The $\mathrm{mScarlet}-\mathrm{I}$ was excited with a $561 \mathrm{~nm}$ laser line $(10 \%$ laser output of WLL), and fluorescence was detected by the hybrid detector from 570 to $620 \mathrm{~nm}$. The images were acquired in photon counting mode, and an accumulation of ten frames per image was taken. Time-gating was active to suppress autofluorescence. Maximum-intensity projections of the images were obtained from $\mathrm{z}$-stack series, capturing $\mathrm{z}$-slices with $1.0 \mu \mathrm{m}$ intervals, and the images were processed using ImageJ.

Reporting Summary. Further information on research design is available in the Nature Research Reporting Summary linked to this article.

\section{Data availability}

All materials generated in this study are freely available upon request from the corresponding author. All data are available in the main text or the supplementary materials. The crystallographic data are available from the RCSB PDB (accession number 6SDG), and the RNA-seq data are available from the NCBI SRA under the project accession number PRJNA554398 (http://www.ncbi.nlm.nih.gov/ bioproject/554398).

\section{Code availability}

The Python script used to calculate the residues in dimer interface is available at http://www.protein.osaka-u.ac.jp/rcsfp/supracryst/suzuki/jpxtal/Katsutani/ InterfaceResidues.py.

Received: 6 September 2019; Accepted: 9 April 2020;

Published online: 15 May 2020 


\section{References}

1. Du, Y. \& Scheres, B. Lateral root formation and the multiple roles of auxin. J. Exp. Bot. 69, 155-167 (2018).

2. Vanneste, S. \& Friml, J. Auxin: a trigger for change in plant development. Cell 136, 1005-1016 (2009).

3. Weijers, D. \& Wagner, D. Transcriptional responses to the auxin hormone. Annu. Rev. Plant Biol. 67, 539-574 (2016).

4. Mutte, S. K. et al. Origin and evolution of the nuclear auxin response system. eLife 7, e33399 (2018).

5. Flores-Sandoval, E. et al. Class C ARFs evolved before the origin of land plants and antagonize differentiation and developmental transitions in Marchantia polymorpha. New Phytol. 218, 1612-1630 (2018).

6. Finet, C., Berne-Dedieu, A., Scutt, C. P. \& Marlétaz, F. Evolution of the ARF gene family in land plants: old domains, new tricks. Mol. Biol. Evol. 30, 45-56 (2013).

7. Ulmasov, T., Hagen, G. \& Guilfoyle, T. J. Activation and repression of transcription by auxin response factors. Proc. Natl Acad. Sci. USA 96, 5844-5849 (1999).

8. Vernoux, T. et al. The auxin signalling network translates dynamic input into robust patterning at the shoot apex. Mol. Syst. Biol. 7, 508 (2011).

9. Piya, S., Shrestha, S. K., Binder, B., Stewart, C. N. Jr. \& Hewezi, T. Proteinprotein interaction and gene co-expression maps of ARFs and Aux/IAAs in Arabidopsis. Front. Plant Sci. 5, 744 (2014).

10. Lavy, M. et al. Constitutive auxin response in Physcomitrella reveals complex interactions between Aux/IAA and ARF proteins. eLife 5, e13325 (2016).

11. Zhao, Z. et al. Hormonal control of the shoot stem-cell niche. Nature 465, 1089-1092 (2010).

12. Boer, D. R. et al. Structural basis for DNA binding specificity by the auxin-dependent ARF transcription factors. Cell 156, 577-589 (2014).

13. Hori, K. et al. Klebsormidium flaccidum genome reveals primary factors for plant terrestrial adaptation. Nat. Commun. 5, 3978 (2014).

14. Bowman, J. L. et al. Insights into land plant evolution garnered from the Marchantia polymorpha genome. Cell 171, 287-304 (2017).

15. Kato, H. et al. Auxin-mediated transcriptional system with a minimal set of components is critical for morphogenesis through the life cycle in Marchantia polymorpha. PLoS Genet. 11, e1005084 (2015).

16. Flores-Sandoval, E., Eklund, D. M. \& Bowman, J. L. A simple auxin transcriptional response system regulates multiple morphogenetic processes in the liverwort Marchantia polymorpha. PLoS Genet. 11, e1005207 (2015).

17. Kato, $\mathrm{H}$. et al. The roles of the sole activator-type auxin response factor in pattern formation of Marchantia polymorpha. Plant Cell Physiol. 58, 1642-1651 (2017)

18. Flores-Sandoval, E., Romani, F. \& Bowman, J. L. Co-expression and transcriptome analysis of Marchantia polymorpha transcription factors supports class C ARFs as independent actors of an ancient auxin regulatory module. Front. Plant Sci. 9, 1345 (2018).

19. Tiwari, S. B., Hagen, G. \& Guilfoyle, T. The roles of auxin response factor domains in auxin-responsive transcription. Plant Cell 15, 533-543 (2003).

20. Choi, H. S., Seo, M. \& Cho, H. T. Two TPL-binding motifs of ARF2 are involved in repression of auxin responses. Front. Plant Sci. 9, 372 (2018).

21. Korasick, D. A. et al. Molecular basis for AUXIN RESPONSE FACTOR protein interaction and the control of auxin response repression. Proc. Natl Acad. Sci. USA 111, 5427-5432 (2014).

22. Nanao, M. H. et al. Structural basis for oligomerization of auxin transcriptional regulators. Nat. Commun. 5, 3617 (2014).

23. Sayou, C. et al. A SAM oligomerization domain shapes the genomic binding landscape of the LEAFY transcription factor. Nat. Commun. 7, 11222 (2016).

24. Ishizaki, K. et al. Development of Gateway binary vector series with four different selection markers for the liverwort Marchantia polymorpha. PLoS ONE 10, e0138876 (2015).

25. Ishizaki, K., Johzuka-Hisatomi, Y., Ishida, S., Iida, S. \& Kohchi, T. Homologous recombination-mediated gene targeting in the liverwort Marchantia polymorpha L. Sci. Rep. 3, 1532 (2013).

26. Sugano, S. S. et al. Efficient CRISPR/Cas9-based genome editing and its application to conditional genetic analysis in Marchantia polymorpha. PLoS ONE 13, e0205117 (2018).

27. Nishihama, R., Ishida, S., Urawa, H., Kamei, Y. \& Kohchi, T. Conditional gene expression/deletion systems for Marchantia polymorpha using its own Heat-Shock promoter and Cre/loxP-mediated site-specific recombination. Plant Cell Physiol. 57, 271-280 (2016).

28. Hohlbein, J., Craggs, T. D. \& Cordes, T. Alternating-laser excitation: single-molecule FRET and beyond. Chem. Soc. Rev. 43, 1156-1171 (2014).

29. Leyser, O. Auxin signaling. Plant Physiol. 176, 465-479 (2018).

30. Rademacher, E. H. et al. A cellular expression map of the Arabidopsis AUXIN RESPONSE FACTOR gene family. Plant J. 68, 597-606 (2011).

31. Chiyoda, S., Ishizaki, K., Kataoka, H., Yamato, K. T. \& Kohchi, T. Direct transformation of the liverwort Marchantia polymorpha L. by particle bombardment using immature thalli developing from spores. Plant Cell Rep. 27, 1467-1473 (2008).
32. Ishizaki, K., Chiyoda, S., Yamato, K. T. \& Kohchi, T. Agrobacterium-mediated transformation of the haploid liverwort Marchantia polymorpha L., an emerging model for plant biology. Plant Cell Physiol. 49, 1084-1091 (2008).

33. Kubota, A., Ishizaki, K., Hosaka, M. \& Kohchi, T. Efficient agrobacteriummediated transformation of the liverwort Marchantia polymorpha using regenerating thalli. Biosci. Biotechnol. Biochem. 77, 167-172 (2013).

34. Zhang, Y., Werling, U. \& Edelmann, W. SLiCE: a novel bacterial cell extract-based DNA cloning method. Nucleic Acids Res. 40, e55 (2012).

35. Juanhuix, J. et al. Developments in optics and performance at BL13-XALOC, the macromolecular crystallography beamline at the ALBA Synchrotron. J. Synchrotron Radiat. 21, 679-689 (2014).

36. Vonrhein, C. et al. Data processing and analysis with the autoPROC toolbox. Acta Crystallogr. D 67, 293-302 (2011).

37. Tickle, I. J. et al. STARANISO (Global Phasing Ltd., 2018); http://staraniso. globalphasing.org/cgi-bin/staraniso.cgi

38. McCoy, A. J. et al. Phaser crystallographic software. J. Appl. Crystallogr. 40, 658-674 (2007).

39. Adams, P. D. et al. PHENIX: a comprehensive Python-based system for macromolecular structure solution. Acta Crystallogr. D 66, 213-221 (2010)

40. Emsley, P., Lohkamp, B., Scott, W. G. \& Cowtan, K. Features and development of Coot. Acta Crystallogr. D 66, 486-501 (2010).

41. Webb, B. \& Sali, A. Comparative protein structure modeling using MODELLER. Curr. Protoc. Bioinformatics 54, 5.6.1-5.6.37 (2016).

42. Kelley, L. A., Mezulis, S., Yates, C. M., Wass, M. N. \& Sternberg, M. J. The Phyre2 web portal for protein modeling, prediction and analysis. Nat. Protoc. 10, 845-858 (2015).

43. van Zundert, G. C. P. et al. The HADDOCK2.2 web server: user-friendly integrative modeling of biomolecular complexes. J. Mol. Biol. 428, 720-725 (2016)

44. Truernit, E. et al. High-resolution whole-mount imaging of three-dimensional tissue organization and gene expression enables the study of phloem development and structure in Arabidopsis. Plant Cell 20, 1494-1503 (2008).

45. Schindelin, J. et al. Fiji: an open-source platform for biological-image analysis. Nat. Methods 9, 676-682 (2012).

46. Raissig, M. T., Gagliardini, V., Jaenisch, J., Grossniklaus, U. \& Baroux, C. Efficient and rapid isolation of early-stage embryos from Arabidopsis thaliana seeds. J. Vis. Exp. 76, e50371 (2013).

47. Trombetta, J. J. et al. Preparation of single-cell RNA-Seq libraries for next generation sequencing. Curr. Protoc. Mol. Biol. 107, 4.22.1-4.22.17 (2014).

48. Kim, D., Langmead, B. \& Salzberg, S. L. HISAT: a fast spliced aligner with low memory requirements. Nat. Methods 12, 357-360 (2015).

49. Li, H. et al. The sequence alignment/map format and SAMtools. Bioinformatics 25, 2078-2079 (2009).

50. Liao, Y., Smyth, G. K. \& Shi, W. featureCounts: an efficient general purpose program for assigning sequence reads to genomic features. Bioinformatics 30, 923-930 (2014).

51. Love, M. I., Huber, W. \& Anders, S. Moderated estimation of fold change and dispersion for RNA-Seq data with DESeq2. Genome Biol. 15, 550 (2014).

52. Freire-Rios, A., Radoeva, T., De Rybel, B., Weijers, D. \& Borst, J. W. FRET-FLIM for visualizing and quantifying protein interactions in live plant cells. Meth. Mol. Biol. 1497, 135-146 (2017).

53. Matsuo, N., Minami, M., Maeda, T. \& Hiratsuka, K. Dual luciferase assay for monitoring transient gene expression in higher plants. Plant Biotechnol. 18, 71-75 (2001).

54. Akagi, T., Ikegami, A. \& Yonemori, K. DkMyb2 wound-induced transcription factor of persimmon (Diospyros kaki Thunb.), contributes to proanthocyanidin regulation. Planta 232, 1045-1059 (2010).

55. van Dijk, M. \& Bonvin, A. M. 3D-DART: a DNA structure modelling server. Nucleic Acids Res. 37, W235-W239 (2009).

56. Kalinin, S. et al. A toolkit and benchmark study for FRET-restrained high-precision structural modeling. Nat. Methods 9, 1218-1225 (2012).

57. Craggs, T. D. et al. Substrate conformational dynamics drive structure-specific recognition of gapped DNA by DNA polymerase. Nucleic Acids Res. 47, 10788-10800 (2019)

58. Farooq, S. \& Hohlbein, J. Camera-based single-molecule FRET detection with improved time resolution. Phys. Chem. Chem. Phys. 17, 27862-27872 (2015).

59. Cordes, T., Vogelsang, J. \& Tinnefeld, P. On the mechanism of Trolox as antiblinking and antibleaching reagent. J. Am. Chem. Soc. 131, 5018-5019 (2009).

60. Rasnik, I., McKinney, S. A. \& Ha, T. Nonblinking and long-lasting single-molecule fluorescence imaging. Nat. Methods 3, 891-893 (2006).

61. Evans, G. W., Hohlbein, J., Craggs, T., Aigrain, L. \& Kapanidis, A. N. Real-time single-molecule studies of the motions of DNA polymerase fingers illuminate DNA synthesis mechanisms. Nucleic Acids Res. 43, 5998-6008 (2015) 


\section{Acknowledgements}

We thank the XALOC staff at the synchrotron ALBA and V. P. Carrillo, Carrasco, S. Kiryu, M. Katayama and L. Olijslager for experimental support, D. Gadella and K. Hiratsuka for providing materials, and O. Leyser for comments in the manuscript. This work was supported by an EMBO Long-term Fellowship (ALTF 415-2016) to H.K., a PhD fellowship from the Graduate School Experimental Plant Sciences to J.H. and D.W., a VICI grant (no. 865.14.001) from the Netherlands Organization for Scientific Research (NWO) to D.W., grants from the Ministry of Economy and Competitiveness of the Spanish Government (nos BIO2016-77883-C2-2-P and FIS2015-72574-EXP) (AEI/ FEDER,EU) to D.R.B., an ALW-open grant (no. ALWOP.402) from the NWO to J.W.B., JSPS/MEXT KAKENHI (grant nos 19K23751 to H.K., 18J12698 to H.S, 19K016166 to Y.Y., $17 \mathrm{H} 06472$ to K.I. $18 \mathrm{H} 04836$ to R.N. and 25113009 , $15 \mathrm{~K} 21758$ and $19 \mathrm{H} 05675$ to T.K.) and SPIRITS 2017 of Kyoto University to R.N.

\section{Author contributions}

H.K., R.N., T.K. and D.W. conceptualized the project. H.K., S.K.M., H.S., I.C., T.R., S.D., M.F., E.H., W.v.d.B. and S.L. conducted the investigation. H.K., S.K.M., I.C. and M.F. conducted the formal analysis. J.H., D.R.B., R.N., T.K. and D.W. supervised the project.
K.I., J.H., R.N., T.K., J.W.B. and D.W. acquired the funding. H.K. and D.W. wrote the original draft of the paper. All authors reviewed and edited the paper.

\section{Competing interests}

The authors declare no competing interests.

\section{Additional information}

Extended data is available for this paper at https://doi.org/10.1038/s41477-020-0662-y. Supplementary information is available for this paper at https://doi.org/10.1038/ s41477-020-0662-y.

Correspondence and requests for materials should be addressed to D.W.

Reprints and permissions information is available at www.nature.com/reprints.

Publisher's note Springer Nature remains neutral with regard to jurisdictional claims in published maps and institutional affiliations.

(c) The Author(s), under exclusive licence to Springer Nature Limited 2020 

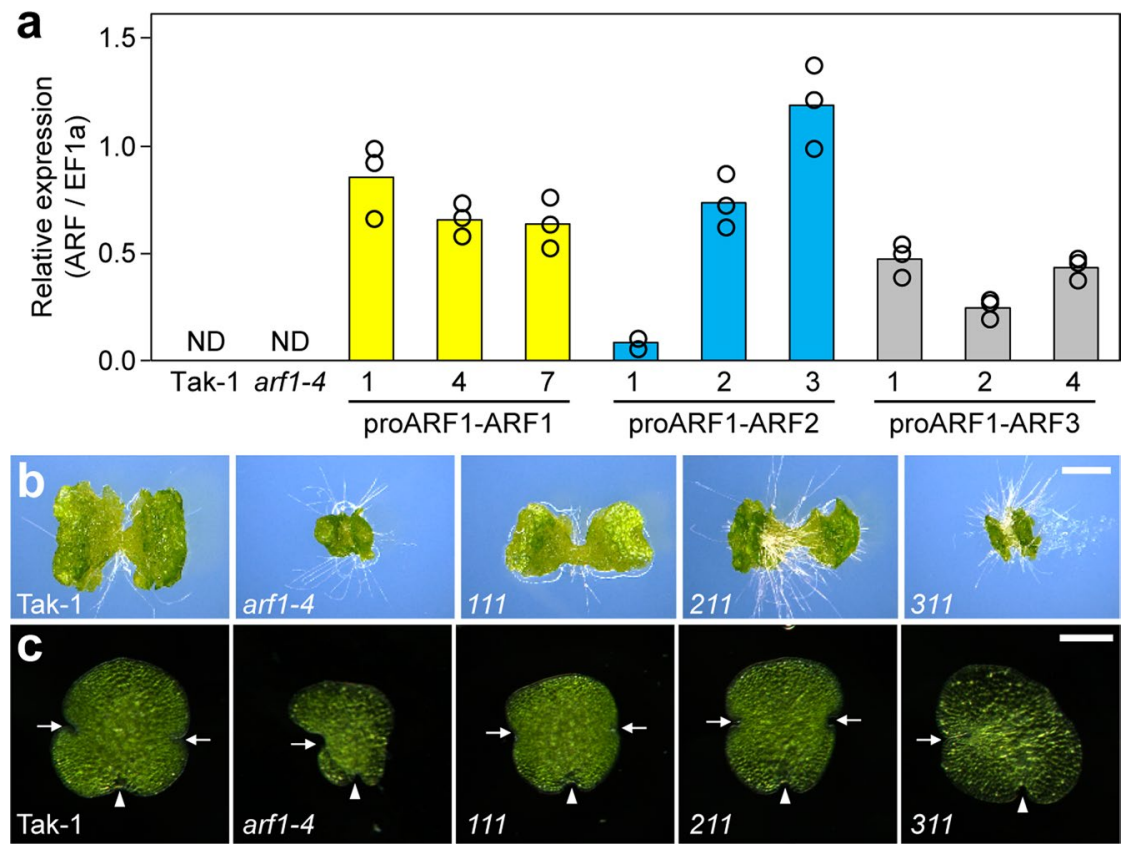

Extended Data Fig. 1 | Transgene expression and phenotypes of DBD swap lines. a, Expression of transgenic transcripts in gemmalings of three independent proARF1-ARF1, proARF1-ARF2 and proARF1-ARF3 lines, measured by qRT-PCR using the common 5'-UTR fragment. Circles indicate each data point for three technical replicates. ND: not detected in Tak-1 wild-type and arf1-4 mutant. $\mathbf{b}$, Ten-day-old gemmalings and $\mathbf{c}$, immature gemmae of

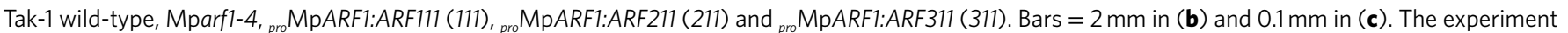
was repeated twice with similar results. 

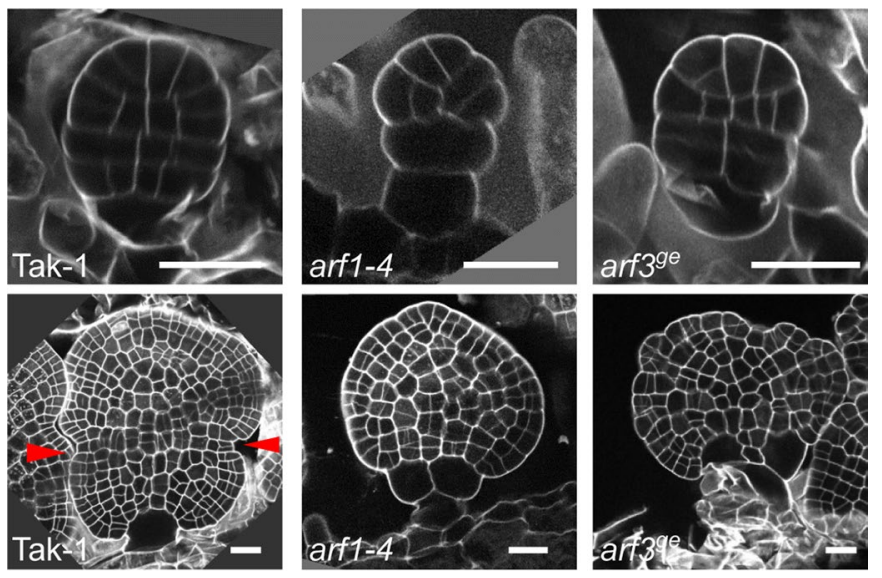

Extended Data Fig. 2 | Gemmae phenotypes in Mparf1 and Mparf3 mutants. Optical medial sections through early- (top row) and late-stage (bottom row) gemmae from Tak-1, Mparf1-4 and Mparf39e1-1 plants. Medial sections were taken from full 3D stacks of gemmae in which cell walls were labeled using mPS-PI staining. The positions of the future apical notches are indicated by red arrowheads in Tak-1. Note that these indentations are missing in both mutants. Bars are $20 \mu \mathrm{m}$ in all panels. The experiment was repeated twice with similar results. 
Downregulated in arf1

(Biological Process)

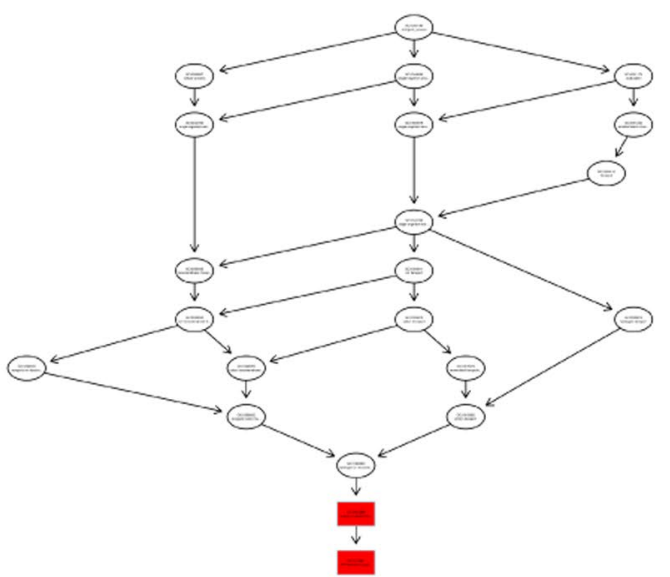

Downregulated in arf1

(Molecular Function

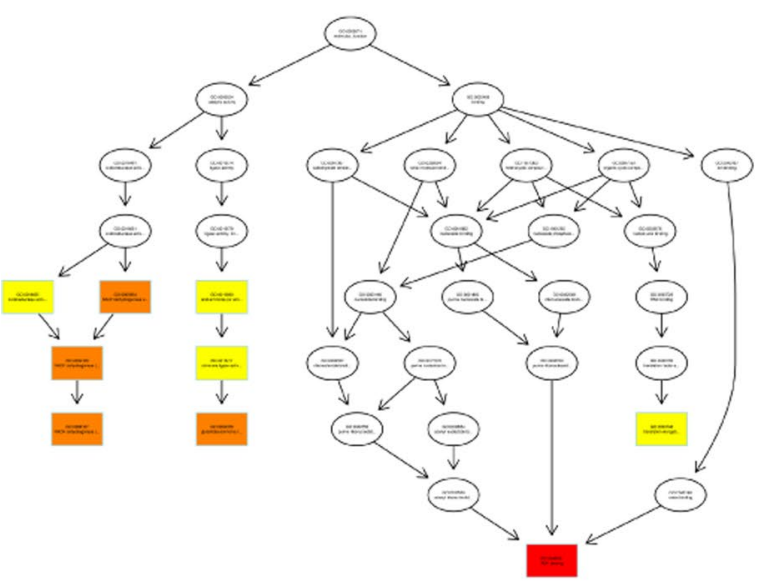

GO.ID

GO:0015988 energy coupled proton transmembrane transport

GO:0015991 ATP hydrolysis coupled proton transport

GO:0043531 ADP binding

G0:0003954 NADH dehydrogenase activity

GO:0004356 glutamate-ammonia ligase activity

GO:0008137 NADH dehydrogenase (ubiquinone) activity

GO:0050136 NADH dehydrogenase (quinone) activity

GO:0016211 ammonia ligase activity

60:0016880 acid-ammonia (or amide) ligase activity

60.0003746 translation elongation factor activity

GO:0016655 oxidoreductase activity, acting on NAD(P)H

$\begin{array}{ll}\text { p-value } & \text { Aspect } \\ 0.0099 & \text { Process } \\ 0.0099 & \text { Process } \\ 0.000068 & \text { Function } \\ 0.0021 & \text { Function } \\ 0.0021 & \text { Function } \\ 0.0021 & \text { Function } \\ 0.0021 & \text { Function } \\ 0.0025 & \text { Function } \\ 0.0025 & \text { Function } \\ 0.0085 & \text { Function } \\ 0.0085 & \text { Function }\end{array}$

Downregulated in arf3

(Biological Process)

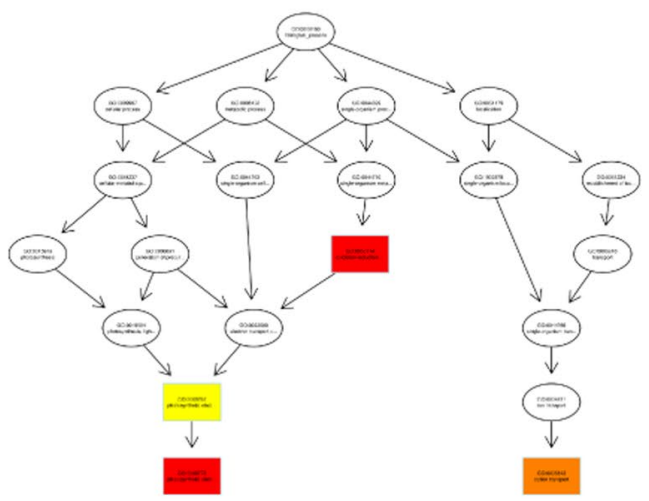

Downregulated in arf3

(Molecular Function

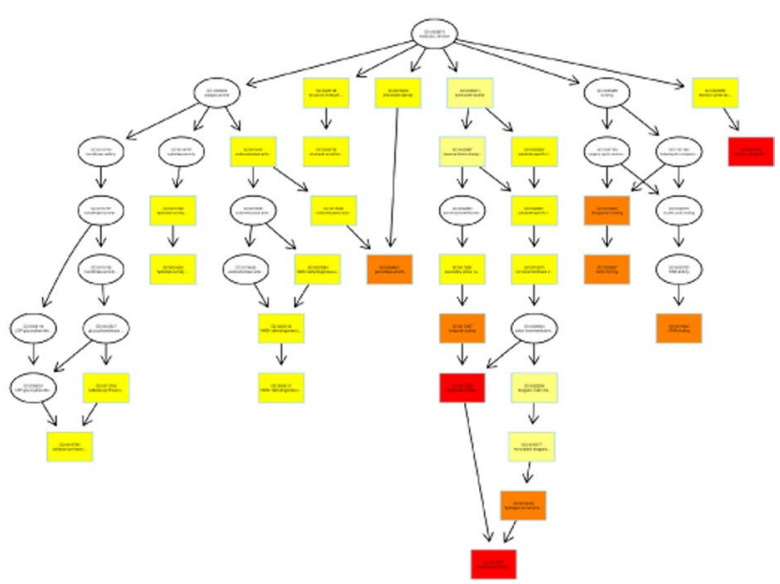

Extended Data Fig. 3 | Gene Ontology analysis of genes misexpressed in arf1 and arf3 gemmae. GO categories 'Biological process' and 'Molecular function' were shown for downregulated genes as directed graphs with least- and most- enriched categories are shown in yellow and red blocks, respectively, as well as in the table underneath the figures. Note the different categories enriched in arf1 and arf3. P-values (table) are derived from a Fisher's exact test. 

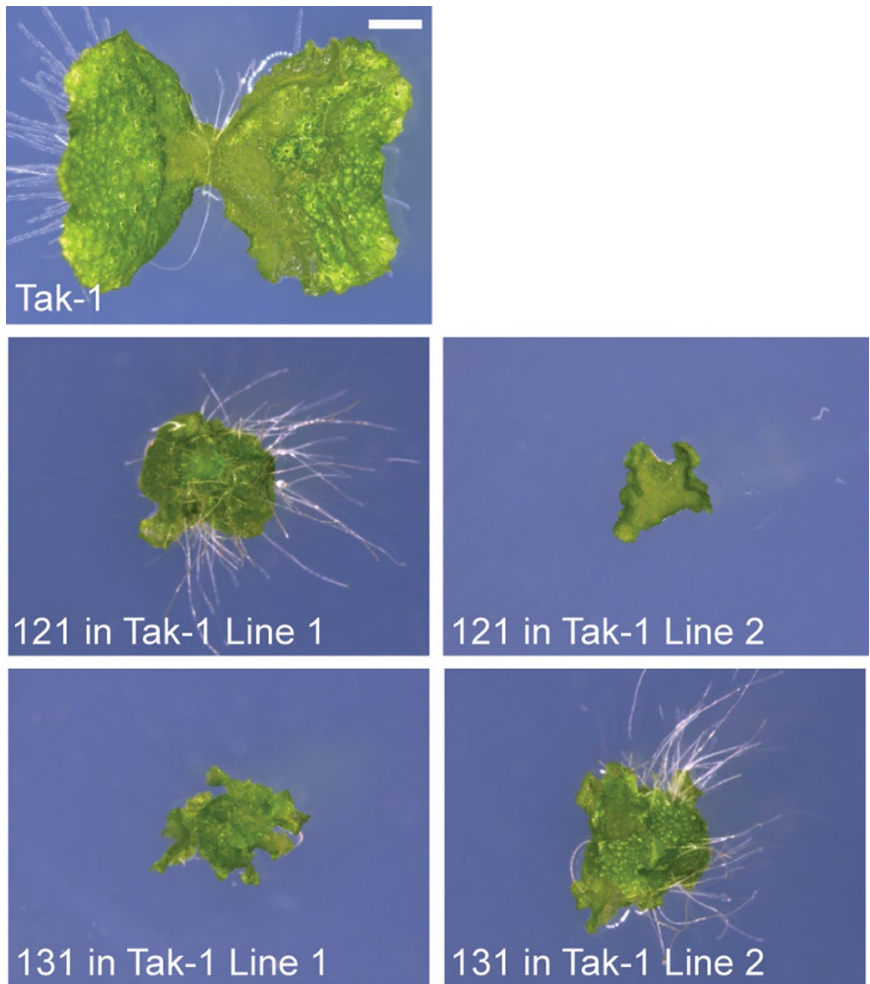

Extended Data Fig. 4 | Phenotypes induced by Middle Region domain swaps in Tak-1 background. Ten-day-old gemmalings of Tak-1 wild-type, and 2

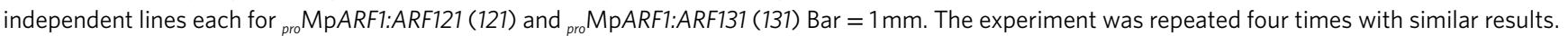



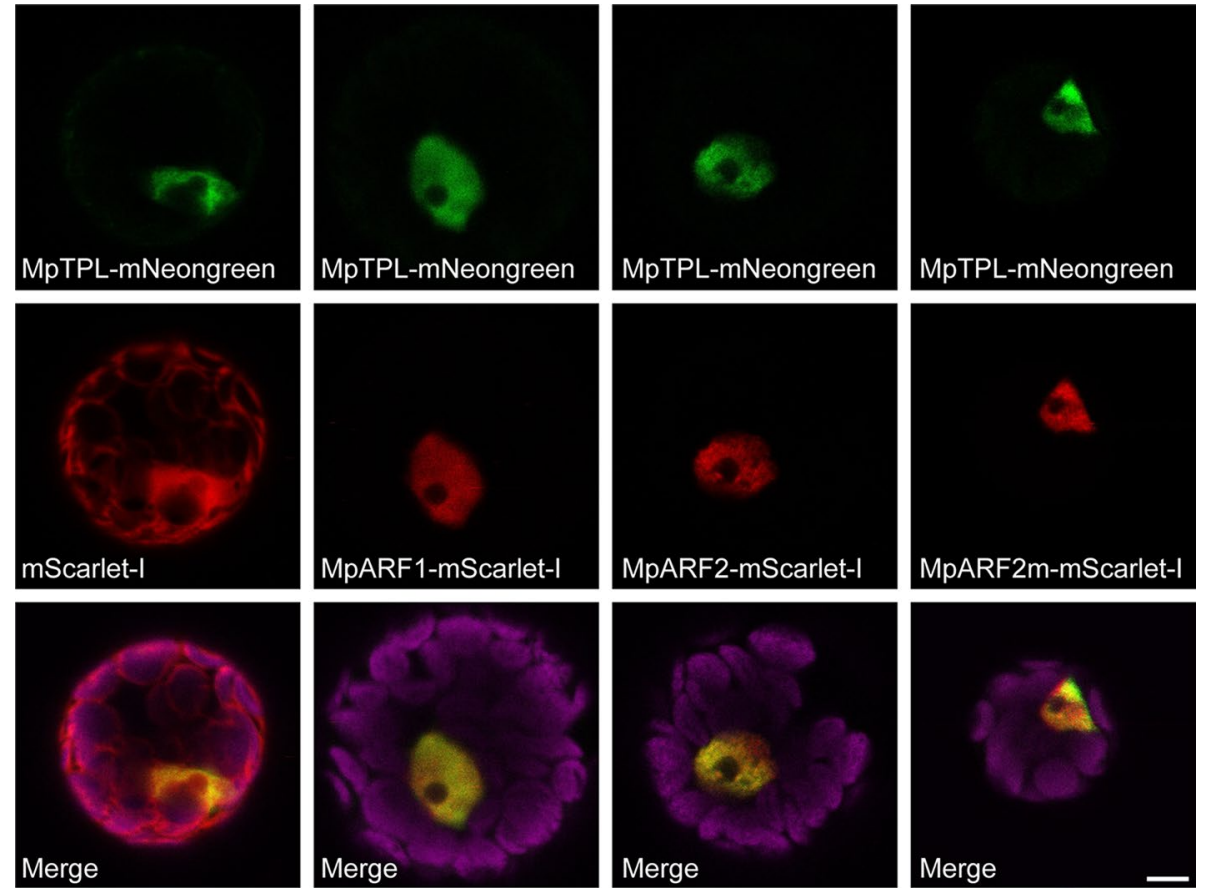

Extended Data Fig. 5 | Expression of MpTPL and MpARFs for FRET-FLIM. Fluorescence of MpTPL-mNeongreen (FRET donor; top row), either co-expressed with fusions of mScarlet-I (FRET acceptor; middle row), to empty vector or with MpARF1-mScarlet-I, MpARF2-mScarlet-I or MpARF2(LFG-AAA)-mScarlet-I. Bottom panel shows overlay of mNeongreen, mScarlet-I and chloroplast autofluorescence signals. Note that all fusion proteins localize to nuclei. Bar is $5 \mu \mathrm{m}$. The experiment was repeated twice with similar results and with 15, 10, 10 and 15 protoplasts (left to right). 


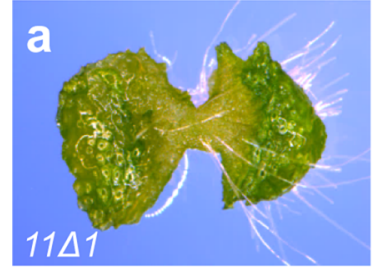

b

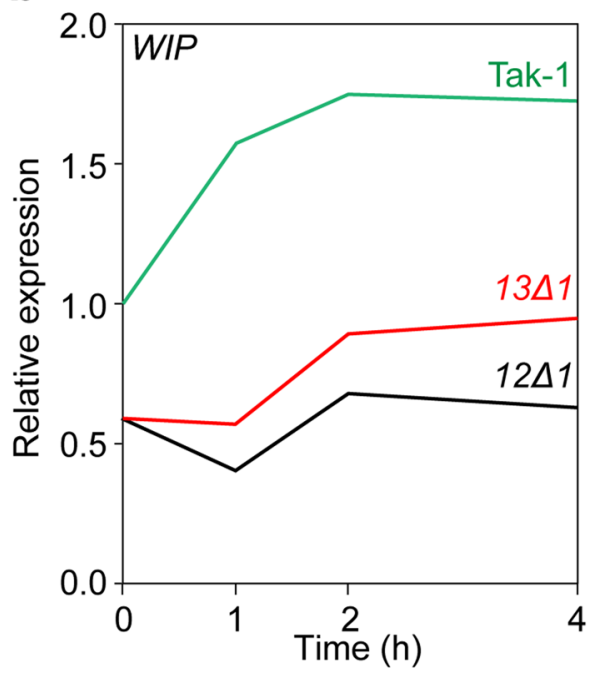

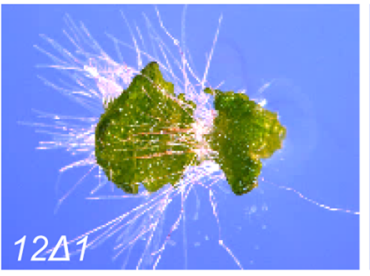

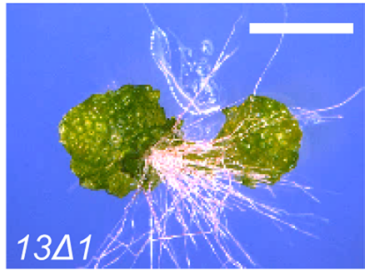

C

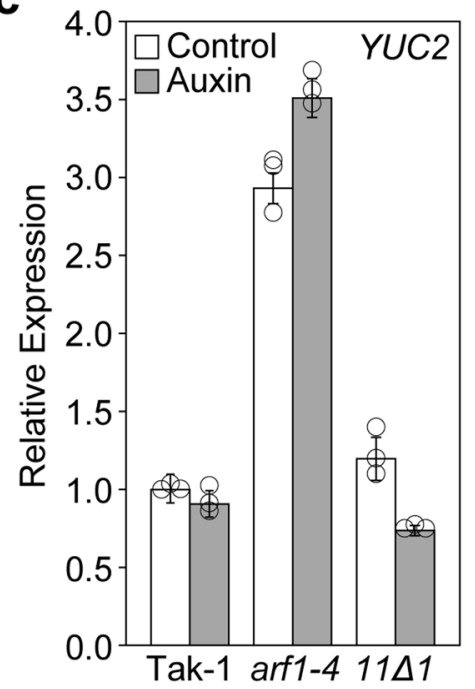

Extended Data Fig. 6 | Biological significance of the MpARF LFG motifs. a, Ten-day-old gemmalings of ${ }_{\text {pro }}$ MpARF1:ARF1141 (11 1), proMpARF1:ARF12 1

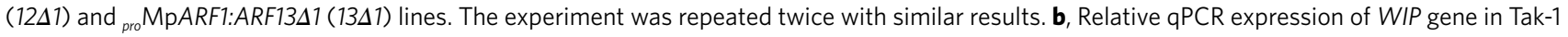

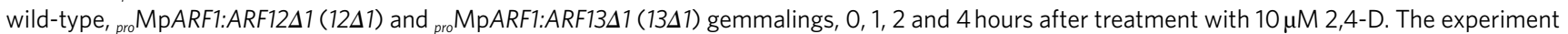
was performed once with three technical replicates. c, Relative qPCR expression of YUC2 gene in Tak-1 wild-type, Mparf1-4 and pro MpARF1:ARF11 1 (11A1) gemmalings, after a 1-hour treatment with control media or with $10 \mu M$ 2,4-D. $n=3$ technical replicates. The experiment was repeated twice with similar results. Bar in $(\mathbf{a})=2 \mathrm{~mm}$ 
MpARF1 PB1

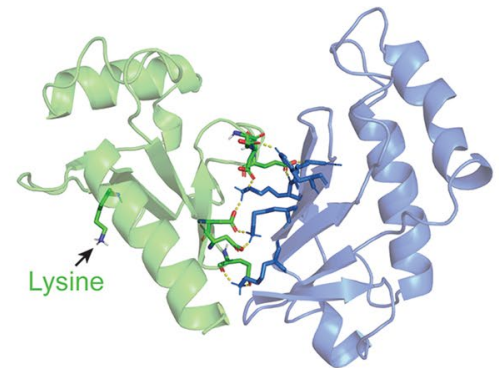

MpIAA PB1
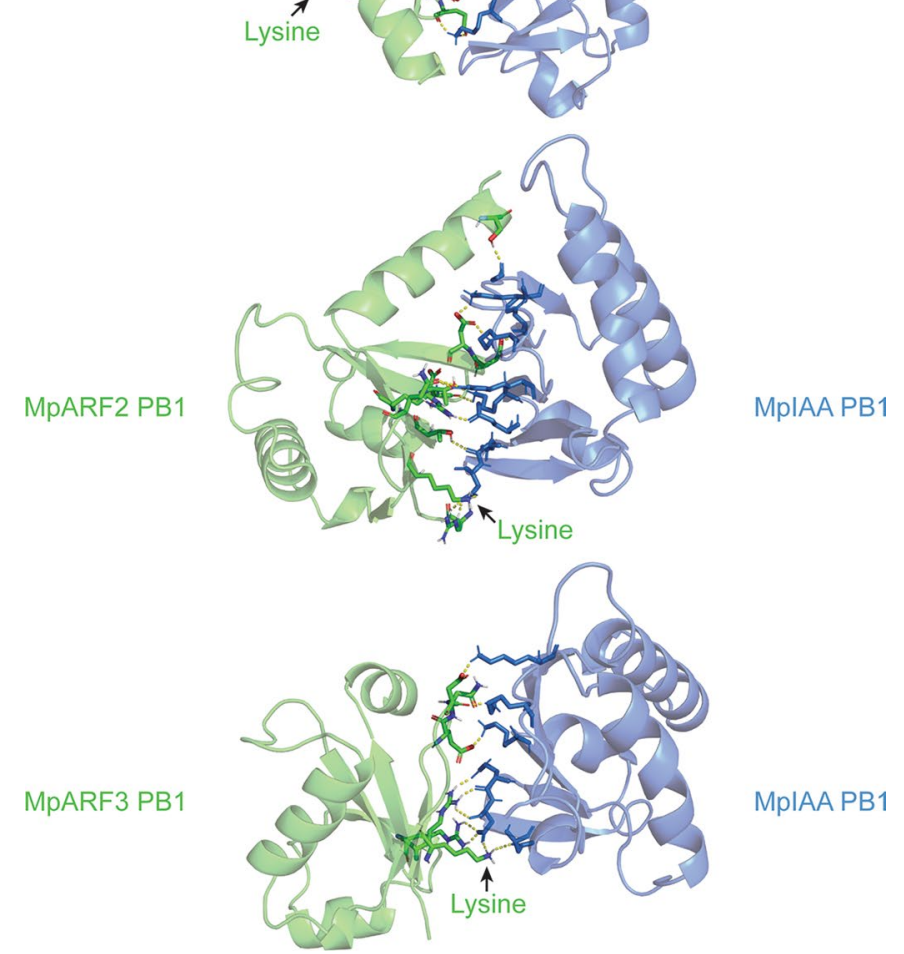

Extended Data Fig. 7 | Docking of MpARF and MpIAA PB1 domains. Best fit structural models of molecular docking of PB1 domains of MpARF1 (top), MpARF2 (middle) and MpARF3 (bottom) with MpIAA. Residues of positive (K, Lysine) and negative (OPCA) faces are indicated as sticks. 


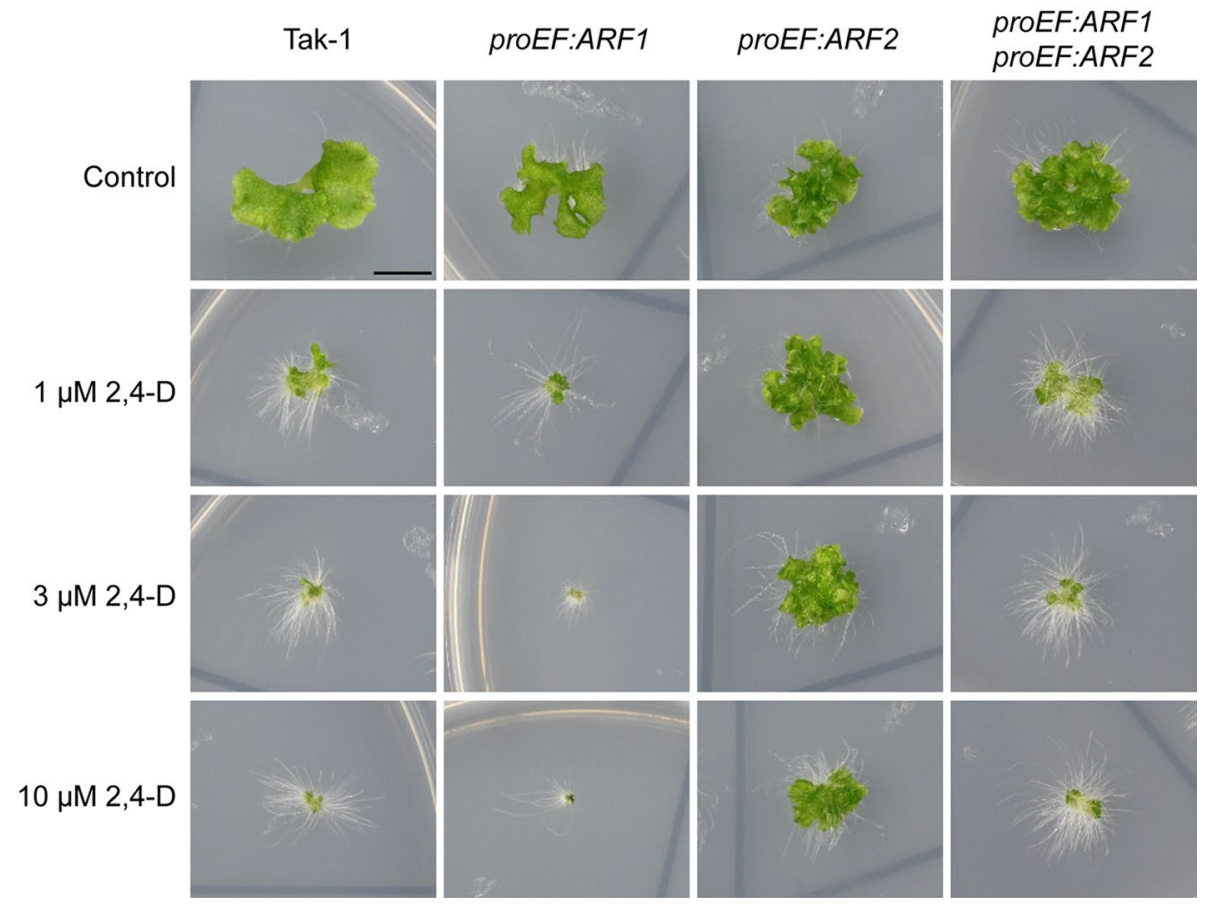

Extended Data Fig. 8 | Antagonistic action of MpARF1 and MpARF2. Fourteen-day-old gemmalings of Tak-1, proF:MpARF1, proF:MpARF2, and ${ }_{\text {pro }} E F: M p A R F 1$ pro EF:MpARF2 transgenic lines grown on control medium, or on media containing 1, 3 or $10 \mu \mathrm{M} 2,4-\mathrm{D}$. The ${ }_{\text {pro }} E F: M p A R F 1_{\text {pro }} E F$ :MpARF2 line was generated by introducing the ${ }_{\text {pro }} E F: M p A R F 1$ cassette into the presented ${ }_{\text {pro }} E F: M p A R F 2$ line. Bar $=1 \mathrm{~cm}$. The experiment was repeated twice with similar results. 
a

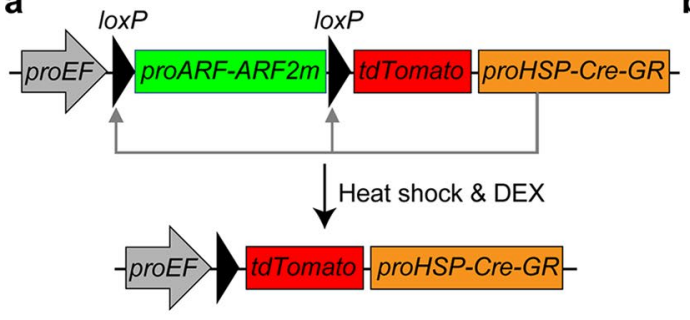

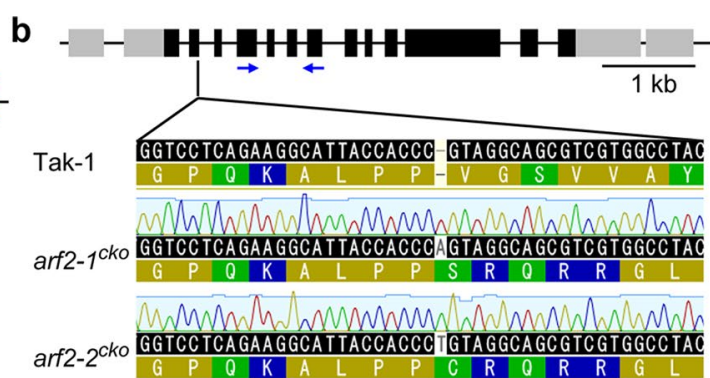

d
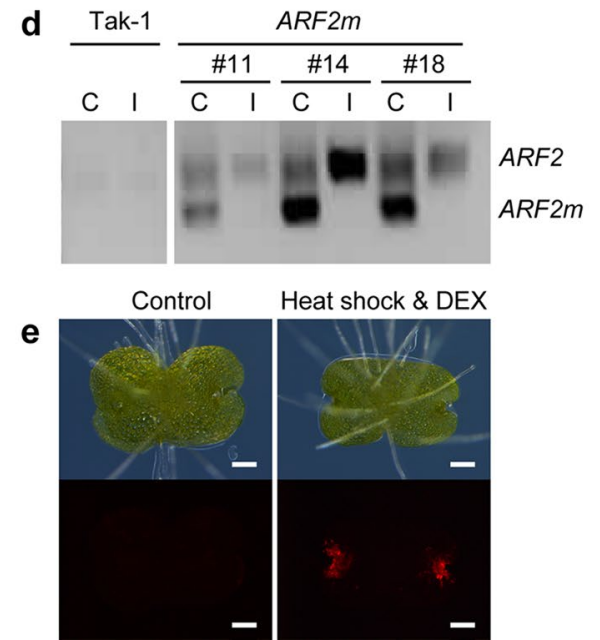

C

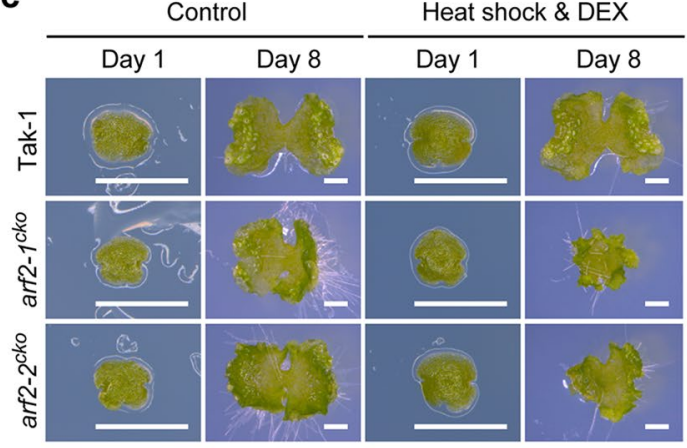

Extended Data Fig. 9 | Conditional MpARF2 inactivation. a, Design of transgene for inducible removal of MpARF2. A CRISPR-resistant MpARF2 version (ARF2m; through engineered silent mutations) is flanked by loxP sites and an upstream EF promoter and a downstream tdTomato-NLS gene, in a construct that also harbors a DEX-inducible Cre-GR version driven from a heat-inducible MpHSP17.8A1 promoter ( ${ }_{\text {pro }} H S P$ ). Following heat shock, and in the presence of DEX, Cre-GR will recombine the two loxP sites and excise the ARF2m locus. Cells in which recombination occurred are marked by ${ }_{\text {pro }}$ F:tdTomato-NLS expression. b, CRISPR/Cas9-induced mutations in the endogenous MpARF2 gene in Mparf2 ${ }^{\text {cko }}$ genetic background. Two different single-base insertions were recovered, generating two independent Mparf $2^{\text {cko }}$ alleles. c, Phenotypes of 8-day-old gemmalings of Tak-1, Mparf2-7cko or Mparf2-2cko lines grown on control medium or with $1 \mu \mathrm{M}$ DEX after a heat shock on day 1 . The experiment was repeated four times with similar results. d, PCR amplicons detecting the endogenous ARF2 (upper band) and ARF2m (lower band) genes in Tak-1 and in 3 independent transgenic lines harboring ARF2m (\#11, \#14, \#18; prior to introduction of the CRISPR/Cas9 construct), either grown on control media, or on $1 \mu \mathrm{M}$ DEX after a heat shock. The experiment was repeated twice with similar results. e, tdTomato expression (lower panels) and appearance (top panels) of 3-day-old Mparf2cko gemmalings grown on control medium or with $1 \mu \mathrm{M}$ DEX after a heat shock on day 1. The experiment was repeated four times with similar results. Bars are $1 \mathrm{~mm}$ in (c) and $0.2 \mathrm{~mm}$ in (e). 
a

5' $\overline{\text { ACTCTTTTTTGTTCGGAAAGGAGACAATCCGTGTG }}$

3' TGAGAAAAAACAGAGCCTTTCCCTCTGTTAGGCACAC

b

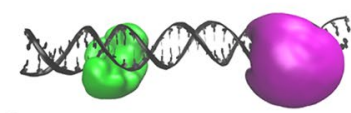

C

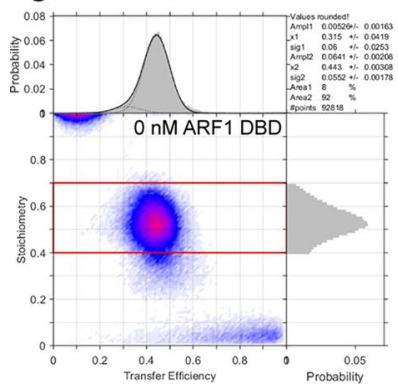

A
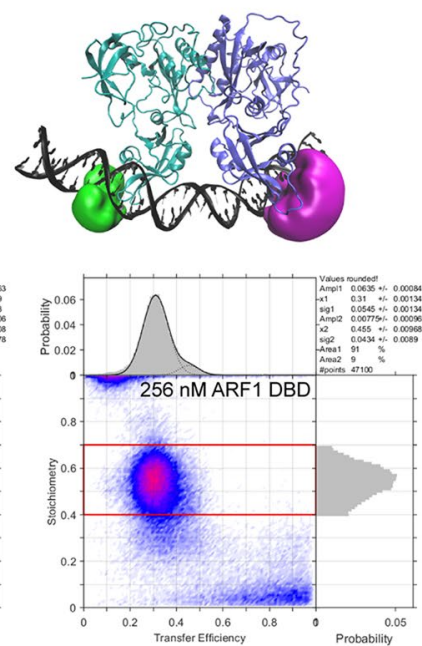

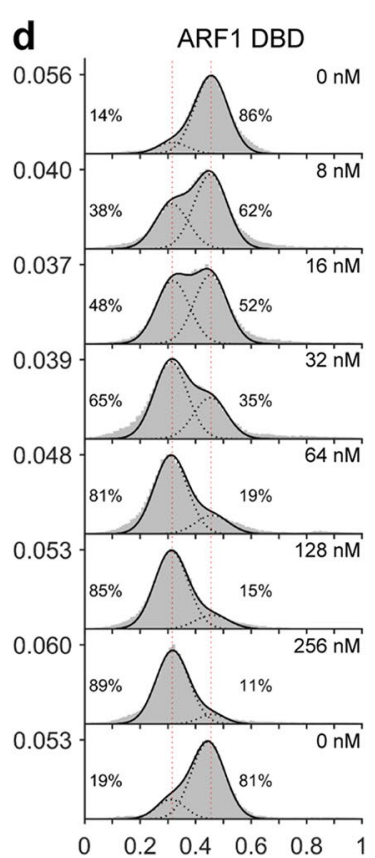

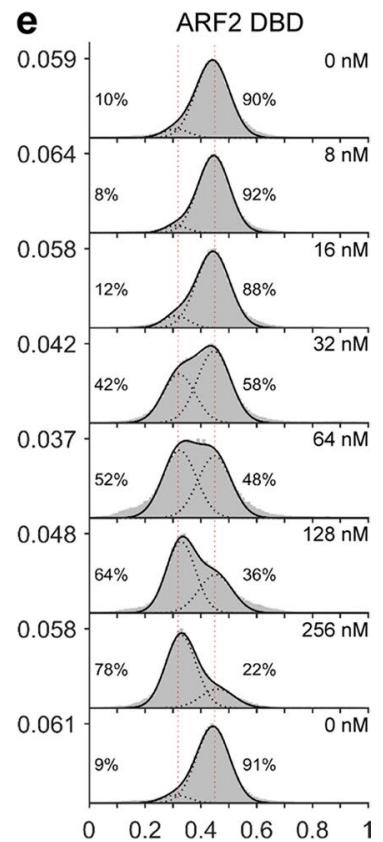

Extended Data Fig. 10 | Single-molecule FRET analysis of ARF-DNA interaction. a, Schematic representation of the ER7 DNA oligonucleotide used in single-molecule FRET experiments, harboring two inverted ARF binding sites (indicated in bold lettering and with a red arrow on top- and bottom-strand, respectively). The bottom-strand was $5^{\prime}$ biotinylated (indicated with an orange hexagon, labeled B) to facilitate immobilization on cover slips.

FRET-compatible Cy3B (green circle) and ATTO647N (magenta circle) labels were attached immediately upstream of each ARF binding site indicated with yellow). b. Simulations of accessible volumes of the Cy3B and ATTO647N dyes on the ER7 oligonucleotide in the absence (left) or presence (right) of MpARF2 DBD (based on the AtARF2-ER7 complex, PDB ID: 6SDG). Note that dye clouds are constrained upon protein binding, predicting reduced FRET efficiency. c, Histograms showing FRET efficiency ( $x$-axis) and Cy3B/ATTO6437N stoichiometry (y-axis) of the immobilized ER7 oligonucleotide incubated without MpARF1 DBD (left) or with $256 \mathrm{nM}$ MpARF1 DBD (right). Each dot represents a single DNA molecule. Note the shift in FRET efficiency induced by protein binding. d,e, Distribution histograms of FRET efficiency ( $\mathrm{x}$-axis) of the labeled and immobilized ER7 oligonucleotide in the presence of increasing concentrations (0-256 nM) of MpARF1 DBD (d) or MpARF2 DBD (e). Each titration series is followed by an incubation without protein to validate recovery to unbound state. Histograms show fits of the two FRET states representing unbound and bound states, and indicate percentages of DNA molecules in each state. 


\section{Reporting Summary}

Nature Research wishes to improve the reproducibility of the work that we publish. This form provides structure for consistency and transparency in reporting. For further information on Nature Research policies, see Authors \& Referees and the Editorial Policy Checklist.

\section{Statistics}

For all statistical analyses, confirm that the following items are present in the figure legend, table legend, main text, or Methods section.

$\mathrm{n} / \mathrm{a}$ | Confirmed

$\square$ \The exact sample size $(n)$ for each experimental group/condition, given as a discrete number and unit of measurement

$\square$ \A statement on whether measurements were taken from distinct samples or whether the same sample was measured repeatedly

The statistical test(s) used AND whether they are one- or two-sided

$\bigotimes$ Only common tests should be described solely by name; describe more complex techniques in the Methods section.

$\square$ \ A description of all covariates tested

$\square$ \ A description of any assumptions or corrections, such as tests of normality and adjustment for multiple comparisons

$\square$ A full description of the statistical parameters including central tendency (e.g. means) or other basic estimates (e.g. regression coefficient)

AND variation (e.g. standard deviation) or associated estimates of uncertainty (e.g. confidence intervals)

$\square$ For null hypothesis testing, the test statistic (e.g. $F, t, r$ ) with confidence intervals, effect sizes, degrees of freedom and $P$ value noted

$\square$ Give P values as exact values whenever suitable.

Х $\square$ For Bayesian analysis, information on the choice of priors and Markov chain Monte Carlo settings

Х $\square$ For hierarchical and complex designs, identification of the appropriate level for tests and full reporting of outcomes

Х $\square$ Estimates of effect sizes (e.g. Cohen's $d$, Pearson's $r$ ), indicating how they were calculated

Our web collection on statistics for biologists contains articles on many of the points above.

\section{Software and code}

Policy information about availability of computer code

Data collection For fluorescence microscopy imaging, LAS-X and LAS-AF were used for Leica SP8 and SP5 systems, respectively. For smFRET assay, Andor SOLIS v4.22.30007.0 was used.

Data analysis For data analysis following programs were used.

Image analysis: ImageJ, Fiji v2.0.0-rc-65/1.52e

Crystallography: Global Phasing AutoPROC, PyMOL v2.4.0a0

Homology modeling: Swissmodel, Modeller v9.17, PyMOL v2.2

RNA-seq: FastQC, TrimGalore v0.5.0, HISAT2 v2.1.0, SAMTOOLS v1.9, DEseq2

FRET-FLIM: SPCImage version 7.1

Accessible Volume simulation: 3D-DART, FRET-restrained positioning and screening (FPS) v1.1, PyMOL v1.3, VMD v1.9.3

SMFRET: twotone v3.1, gaussFigTools v1.0, MATLABR2017b

Docking: HADDOCK2

GO analysis: PlantRegMap

For manuscripts utilizing custom algorithms or software that are central to the research but not yet described in published literature, software must be made available to editors/reviewers. We strongly encourage code deposition in a community repository (e.g. GitHub). See the Nature Research guidelines for submitting code \& software for further information.

\section{Data}

Policy information about availability of data

All manuscripts must include a data availability statement. This statement should provide the following information, where applicable:

- Accession codes, unique identifiers, or web links for publicly available datasets

- A list of figures that have associated raw data

- A description of any restrictions on data availability 


\section{Field-specific reporting}

Please select the one below that is the best fit for your research. If you are not sure, read the appropriate sections before making your selection. \Life sciences

\section{Life sciences study design}

All studies must disclose on these points even when the disclosure is negative.

\begin{tabular}{|c|c|}
\hline Sample size & Sample sizes were determined based on the authors' experience and conventions in the field \\
\hline Data exclusions & No data were excluded from the analysis. \\
\hline Replication & $\begin{array}{l}\text { All measurements were performed multiple times. For transgenic plants, more than three genetically independent lines were tested, and at } \\
\text { least } 10 \text { individuals were used for statistical analysis. All attempts at replication were successful. }\end{array}$ \\
\hline Randomization & No randomization were applied in this study. Samples were grouped based on their genotype, and presence of chemical treatment. \\
\hline Blinding & $\begin{array}{l}\text { Investigators were not blinded to group allocation since there were no cases of minute differences that needed blinding. Where possible, } \\
\text { objective quantification was performed. }\end{array}$ \\
\hline
\end{tabular}

\section{Reporting for specific materials, systems and methods}

We require information from authors about some types of materials, experimental systems and methods used in many studies. Here, indicate whether each material, system or method listed is relevant to your study. If you are not sure if a list item applies to your research, read the appropriate section before selecting a response.

\begin{tabular}{|c|c|c|c|}
\hline \multicolumn{2}{|r|}{ Materials \& experimental systems } & \multicolumn{2}{|c|}{ Methods } \\
\hline $\mathrm{n} / \mathrm{a}$ & Involved in the study & $\mathrm{n} / \mathrm{a}$ & Involved in the study \\
\hline Х & Antibodies & Х & ChIP-seq \\
\hline$\bigotimes$ & $\square$ Eukaryotic cell lines & 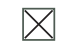 & $\square$ Flow cytometry \\
\hline Х & Palaeontology & Х & MRI-based neuroimaging \\
\hline$\bigotimes$ & $\square$ Animals and other organisms & & \\
\hline$\bigotimes$ & $\square$ Human research participants & & \\
\hline Х & $\square$ Clinical data & & \\
\hline
\end{tabular}

